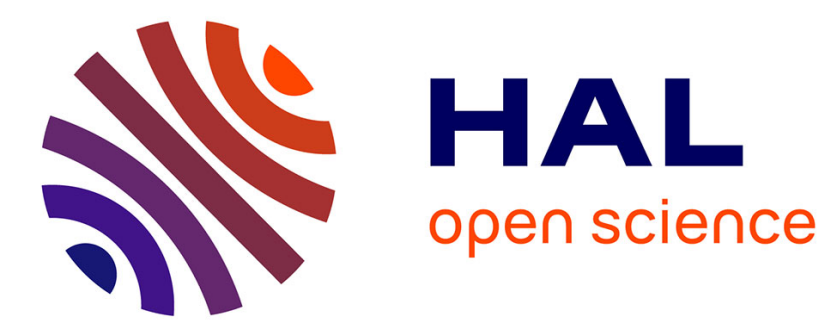

\title{
Commande des feux de signalisation par réseaux de Petri hybrides
}

Cherif Tolba, Dimitri Lefebvre, Philippe Thomas, Abdellah Elmoudni

\section{To cite this version:}

Cherif Tolba, Dimitri Lefebvre, Philippe Thomas, Abdellah Elmoudni. Commande des feux de signalisation par réseaux de Petri hybrides. Journal Européen des Systèmes Automatisés (JESA), 2008, 42 (5), pp.579-612. 10.3166/jesa.42.579-612 . hal-00292749

\section{HAL Id: hal-00292749 \\ https://hal.science/hal-00292749}

Submitted on 2 Jul 2008

HAL is a multi-disciplinary open access archive for the deposit and dissemination of scientific research documents, whether they are published or not. The documents may come from teaching and research institutions in France or abroad, or from public or private research centers.
L'archive ouverte pluridisciplinaire HAL, est destinée au dépôt et à la diffusion de documents scientifiques de niveau recherche, publiés ou non, émanant des établissements d'enseignement et de recherche français ou étrangers, des laboratoires publics ou privés. 
C. Tolba, D. Lefebvre, P. Thomas, A. ElMoudni, « Commande des feux de signalisation par réseaux de Petri hybrides », JESA, Vol.42, n5, pp 579-612, 2008.

\title{
Commande des feux de signalisation par réseaux de Petri hybrides
}

\author{
Chérif Tolba - Dimitri Lefebvre* - Philippe Thomas**- \\ Abdellah EIMoudni**** \\ cherif.tolba@laposte.net \\ *Laboratoire GREAH, Université du Havre, 25 rue P. Lebon-BP1123-76063 LE \\ HAVRE, France \\ dimitri.lefebvre@univ-lehavre.fr \\ **Centre de Recherche en Automatique de Nancy (CRAN-UMR 7039), Nancy- \\ University, CNRS, ENSTIB 27 rue du merle blanc, B.P. 1041, 88051 Epinal cedex 9 \\ France \\ philippe.thomas@cran.uhp-nancy.fr \\ ***Laboratoire systèmes et Transport, Université de Belfort-Montbéliard, 90010 \\ Belfort Cedex, France \\ abdellah.elmoudni@utbm.fr
}

RÉSUMÉ. Cet article traite de la commande des feux de carrefour à l'aide de réseaux de Petri hybrides. Après avoir modélisé la dynamique hétérogène du trafic, les caractéristiques non linéaires du modèle obtenu sont mises en évidence. La linéarisation de ce modèle par l'approche multi - modèles permet de mettre en ouvre des commandes événementielles basées sur la détection de certains événements internes. Les performances de ces commandes sont comparées aux performances obtenues à l'aide de commandes usuelles utilisées pour la synchronisation des feux des carrefours isolés. Enfin, l'approche a été étendue à la régulation du trafic dans un réseau de plusieurs carrefours.

ABSTRACT. This article deals with traffic lights control by using hybrid Petri nets. The modelling of traffic heterogeneous dynamic leads to non linear characteristics. Linearization of this model by means of multi - models made it possible to implement discrete event controllers based on thresholds detection. Performances of the resulting controllers are compared with usual controllers used to synchronization of traffic lights in isolated crossroad. This approach was also extended to traffic control in large scale networks.

MOTS-CLÉS : régulation du trafic, Réseau de Petri hybride, système non linéaire, approche multi - modèle, commande événementielle.

KEYWORDS: traffic control, hybrid Petri net, non linear system, multi - model approach, event control. 


\section{Introduction}

Depuis la fin de la deuxième guerre mondiale, les villes n'ont pas cessé de s'étendre. Afin d'absorber les flux de personnes et de marchandises, les voies de circulation se sont multipliées jusqu'à représenter, presque, la moitié de la superficie totale des zones urbaines. Cet accroissement du nombre de voies de circulation n'est cependant pas suffisant, ce qui implique l'apparition de phénomènes de congestion et de files d'attente, et dans le pire des cas, provoquent des accidents. De manière générale, la congestion conduit à la dégradation de la qualité de service des infrastructures routières.

Le processus d'évolution du trafic peut être assimilé à un système dynamique hybride dont l'évolution des files d'attente est décrite par une partie continue et l'occurrence d'évènements par une partie discrète. Ceci s'explique par le fait que l'approche hybride reflète la réalité d'évolution des systèmes de transport. En terme de modélisation, la partie continue décrivant l'évolution instantanée du système est représentée par un ensemble de variables d'état qui évoluent selon des équations différentielles validées dans des domaines temporels spécifiés. La partie discrète est décrite par une suite d'états discrets dépendant d'une séquence d'événements (internes ou externes). La synthèse des lois de commande des systèmes hybrides se situe au cœur des recherches menées actuellement par de nombreux scientifiques (Antsaklis et Koutsoukos, 2002).

En ce qui concerne la gestion du trafic pour une utilisation optimale des infrastructures disponibles, de nombreuses stratégies de régulation des feux de signalisation ont été mises en place. Ces stratégies reposent principalement sur l'estimation des temps d'attente et des longueurs des files d'attente aux intersections. Ces paramètres permettent d'évaluer la qualité de service des intersections à feux. L'approche traditionnelle dans la commande des feux utilise des plans de signalisation à période fixe pour lesquels les durées des feux sont prédéterminées. Dans ce cas les paramètres essentiels de la commande, comme la durée du cycle, le partage du vert et les décalages sont calculés hors ligne, à partir d'un historique des données (Cohen, 1990). Bien que la réalisation de cette stratégie soit basée sur des données recueillies sur le terrain, la gestion du trafic reste éloignée d'une exploitation optimale des ressources car on ne parvient pas à suivre les fluctuations de la demande.

Depuis les avancées significatives dans les domaines de l'informatique et de la télécommunication, le calcul des paramètres de la commande des feux se fait en temps réel à partir de mesures transmises par des boucles électromagnétiques ou des caméras numériques. Ces stratégies de commandes adaptatives régulent l'état des feux en fonction de la présence ou non de véhicules à l'intersection. De nombreux simulateurs sont ainsi développés pour la régulation des réseaux urbains : «SCOOT (Hunt et al., 1981 ; Hansen et al., 2000) »; «PRODYN (Henry, 1983 ; Khoudour et al., 1991) » ; «OPAC (Gartner et al., 1991) »; «SCATS (Sims et Finaly, 1984 ; Taylor et Abdel-Rahim, 1998) »; «CRONOS (Boillot, 2002)». 
L'exploitation du potentiel des réseaux de Petri (RdP) (David et Alla, 1992) dans la régulation des feux de trafic a été initiée par Jensen (1992) en modélisant les feux de signalisation par un RdP coloré. Wang et al., (1993) ont utilisé les RdP pour la commande et l'évaluation des performances d'une intersection isolée en se basant sur le simulateur SIMNET (Simulation Nets). DiCesare et al. (1994) ont proposé une approche modulaire pour l'évaluation et la commande de six intersections adjacentes. Ces résultats étaient obtenus avec le simulateur POSES (Predicate Transition Net Oriented Simulation). Gallego et al., (1996) ont développé un contrôleur des feux de signalisation à base de réseaux de Petri interprétés (David et Alla, 1992). Le contrôleur proposé a pour rôle de traduire certaines contraintes imposées permettant de garantir le bon fonctionnement d'un carrefour à feux. Récemment, les RdP hybrides (Le Bail et al., 1991) ont aussi été appliqués avec succès pour la modélisation et la commande des systèmes de transport (Di Febrarro et al., 2004).

Dans cet article nous présentons un système de commande des feux de signalisation basé sur les RdP hybrides (RdPH) qui modélisent les dynamiques hétérogènes des véhicules dans le carrefour. La circulation des véhicules est caractérisée par un comportement continu sur les routes et par un comportement discret au niveau des croisements routiers. Ces deux comportements sont respectivement modélisés par un RdP continu à vitesses variables (RdPCV) (Tolba et al., 2001) et un RdP Temporisé (RdPT) (Tolba et al., 2003). Après avoir modélisé l'écoulement du trafic ainsi que les interactions événementielles résultant du changement de l'état des feux de signalisation, le comportement non linéaire du trafic est décomposé en multi-modèles linéaires. La détection des limites des domaines de validité de ces modèles permet d'évaluer l'état du trafic et par conséquent de commuter ou non le feu.

Cet article est organisé en six sections. Dans la section 2, nous décrivons la topologie d'un carrefour isolé et ensuite nous présentons son modèle par RdP hybride. Une fois le modèle établi, nous essayons d'élargir cette approche à la modélisation d'un ensemble de carrefours interconnectés. L'approche multi modèle et la commande par rupture de seuils sont présentées respectivement dans les sections 3 et 4 . Des simulations pour l'analyse et la comparaison de la commande par rupture de seuils appliquée à un carrefour isolé et étendue à un réseau complexe font l'objet de la section 5. Enfin, des conclusions et des perspectives de recherches sont exposées dans la section 6 .

\section{Modèle par RdP hybride du trafic}

Dans cette section on traite un exemple d'application des réseaux de Petri dans les systèmes de transport.

\subsection{Topologie d'un carrefour à feux}

Le système étudié est un carrefour à feux isolé qui comporte deux voies $\left(L_{1}, L_{2}\right)$ avec deux feux de signalisation $\left(T^{*}{ }_{1}, T^{*}{ }_{2}\right)$ implantés à l'extrémité du carrefour, Figure 1. 
Les voies du carrefour de la Figure 1 sont caractérisées par un taux d'arrivée moyen égal à $\mu_{1}$ et $\mu_{2}$. Des boucles de détection de véhicules $(B 1, B 2)$ sont implantées, respectivement à l'entrée des voies $L_{l}$ et $L_{2}$, à une distance $\Delta_{l}$ et $\Delta_{2}$ de la ligne d'arrêt. Chaque voie du carrefour est de capacité limitée égale à $c$. La vitesse libre $v_{\text {free }}$, correspond à la vitesse maximale que peut atteindre un véhicule empruntant le carrefour. Le trafic s'écoule dans deux directions: Ouest-Est $(O-E)$ et Nord-Sud $(N-S)$. Par souci de simplicité, les voies de circulation sont supposées être à sens unique et la prise en compte des mouvements de tournes à gauche et à droite est exclue de notre étude. De plus le feu a deux états « vert» et « rouge ». La durée du feu « orange » est ajoutée à celle du feu « rouge ».

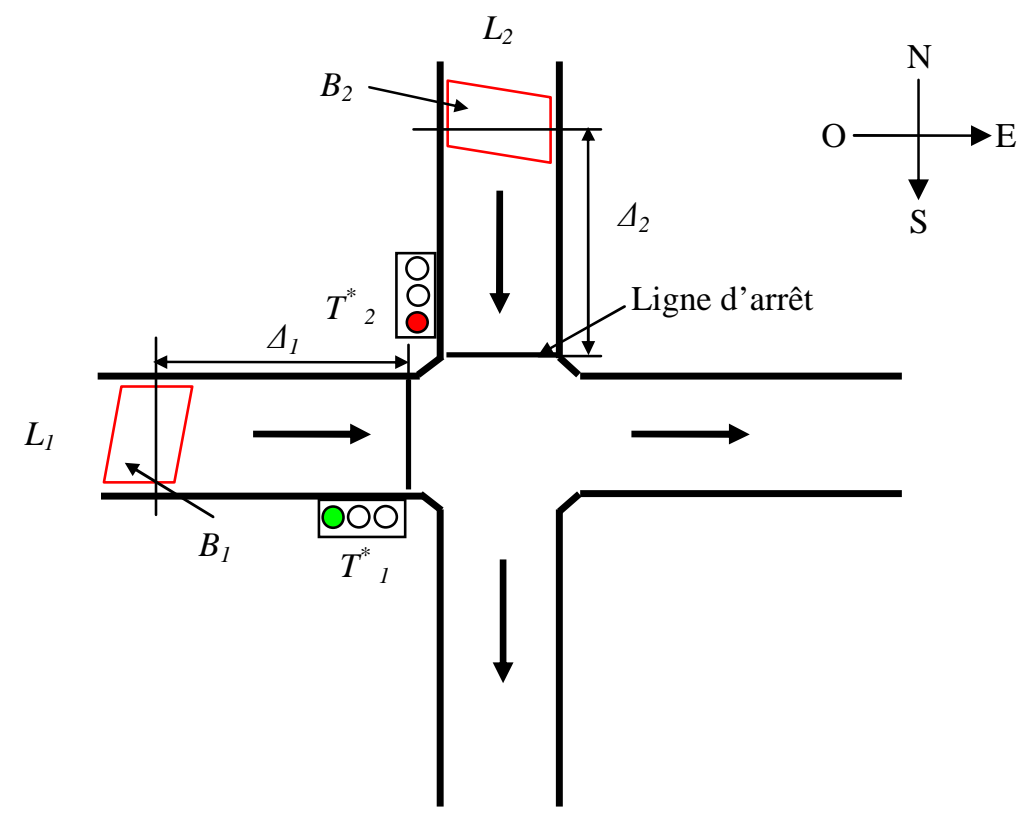

Figure 1. Description d'un carrefour isolé.

\subsection{RdP hybrides}

Les réseaux de Petri hybrides (RdPH) sont une extension des RdP (Le Bail et al., 1991). Dans les RdPH, la représentation des systèmes à comportements hétérogènes est décrite par deux parties distinctes : une partie continue dont le comportement est décrit par un RdP continu et une partie discrète qui est modélisée par un RdP temporisé (à temporisation déterministe ou stochastique). Le marquage d'une place continue est représenté par un nombre réel positif. En revanche, le marquage d'une place discrète est représenté par un nombre entier non négatif. Le franchissement d'une transition discrète peut entraîner une modification sur le marquage du RdP 
continu et vice versa. L'intersection de la figure 1 est un système hybride (Lei et Ozguner, 2001) dans lequel l'écoulement du trafic sur les voies peut être représenté par un RdP continu et le changement des états de feu par un RdP discret (Di Febbraro et al., 2004) (Tolba et al., 2004). Grâce à une approche de modélisation modulaire qui consiste à décomposer le fonctionnement du carrefour en deux modules principaux (feux de signalisation et écoulement du trafic), nous allons présenter le modèle par RdPH du carrefour à feux.

\subsection{Modèle par RdP des sections de route}

Dans ce paragraphe, nous présentons le modèle par RdPCV (Tolba et al., 2001) permettant de modéliser l'écoulement du trafic sur les voies du carrefour, Figure 2.

Avant d'aborder la méthode de modélisation du trafic, nous allons rappeler les principes de base d'un RdPCV. Lorsque les dynamiques d'un système physique sont nombreuses, sa modélisation par un RdP discret nécessite un nombre de marquages accessibles très grand. Les RdP continus ont été introduits pour éviter l'explosion combinatoire du nombre d'états accessibles. Cette extension de RdP permet, par ailleurs, d'élargir le cadre des RdP aux systèmes qui ne sont pas modélisables par des RdP ordinaires (tels que l'écoulement d'un fluide dans un réservoir, la production des produits par lot). Contrairement aux RdP ordinaires ou temporisés (David et Alla, 1992), le nombre de marques dans les RdP continus est un nombre réel positif. Le franchissement s'effectue comme un flot continu en introduisant la notion de vitesse traduite par le nombre de marques franchies pendant une unité de temps. Le franchissement d'une transition dans les RdP continus suit dans le cas général la loi régissant la dynamique du système étudié.

Dans le cas du transport terrestre, chaque section de voirie est représentée par une place $P_{i}$ continue (représentée par un double cercle) dans laquelle le marquage $m_{i}$ représente le nombre de véhicules présents sur la section. Chaque place $P_{i}$ est associée à une place $P^{\prime}{ }_{i}$ continue pour représenter la limitation de capacité de la place $P_{i}$ qui traduit la limitation de capacité sur la section de voirie considérée. Le nombre d'emplacements non occupés sur une section du carrefour est représenté par le marquage $m_{i}{ }_{i}$ de la place $P^{\prime}{ }_{i}$. L'entrée de chaque section est modélisée par une transition $T_{j-1}$ (représentée par un rectangle) dont la fréquence de franchissement maximale est représentée par $q_{\max j-1}=1 / \theta^{i n}\left(\theta^{i n}\right.$ représente le temps d'arrivée moyen d'un véhicule sur la voie). De même, la sortie d'une section est modélisée par une transition $T_{j}$ dont la fréquence maximale de franchissement est l'inverse du temps moyen nécessaire pour qu'un véhicule traverse la section $q_{\max j}=1 / \theta^{\text {out }}$. Le nombre moyen de véhicules pouvant entrer ou traverser simultanément le carrefour est représenté par le marquage $a_{j}$ de la place $P_{j}$. 
C. Tolba, D. Lefebvre, P. Thomas, A. ElMoudni, « Commande des feux de signalisation par réseaux de Petri hybrides », JESA, Vol.42, n5, pp 579-612, 2008.

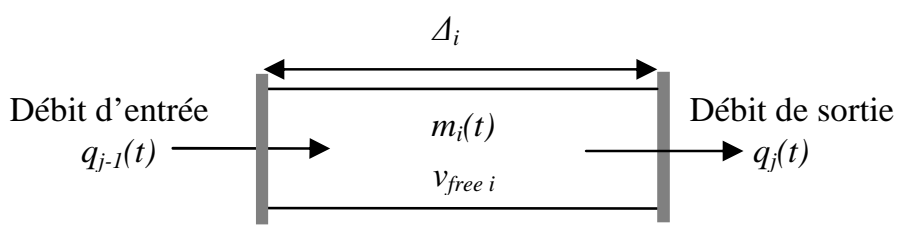

Figure 2. Section de voirie.

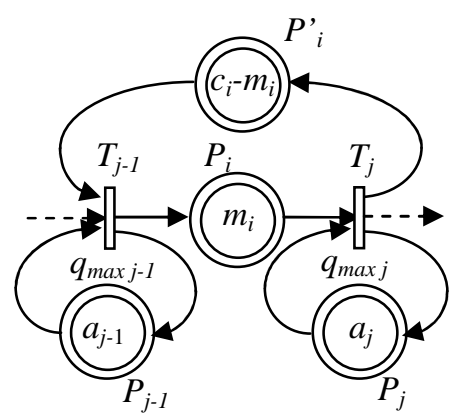

Figure 3. Modèle par RdPCV d'une section de voirie.

Il convient de remarquer que la somme des marquages des places $P_{i}$ et $P^{\prime}$ (i.e. $m_{i}+m_{i}{ }_{i}$ ) est un invariant de marquage. Il en est de même pour le marquage des places $P_{j}$ (i.e. $m_{j}$ ) qui reste constant au cours du temps:

$$
\left\{\begin{array}{lc}
m_{i}(t)+m_{i}^{\prime}(t)=c_{i} & \forall t \geq 0, \quad i=1,2 \\
m_{j}(t)=a_{j} & \forall t \geq 0, \quad j=1, \ldots, 4
\end{array}\right.
$$

où $c_{i}$ représente la capacité de chaque voie.

Conformément au principe d'évolution d'un RdPCV (David et Alla, 1992), les expressions mathématiques des vitesses de franchissement $q_{j-1}(t)$ et $q_{j}(t)$ des transitions $T_{j-1}$ et $T_{j}$ sont données par $^{1}$ :

$$
\begin{aligned}
& q_{j-1}(t)=q_{\max j-1} \min \left(a_{j-1}, c_{i}-m_{i}(t)\right) \\
& q_{j}(t)=q_{\max j} \min \left(a_{j}, m_{i}(t)\right)
\end{aligned}
$$

Lorsque les conditions de validation des transitions $T_{j-1}$ et $T_{j}$ sont remplies (i.e., arrivée et départ de véhicules), l'évolution du marquage $m_{i}$ de la place $P_{i}$ est régie par l'équation suivante :

\footnotetext{
${ }^{1}$ La vitesse de franchissement d'une transition à l'instant $t$ dépend continûment du marquage des places en amont de cette transition (David et Alla, 1992).
} 


$$
\frac{d m_{i}(t)}{d t}=q_{j-1}(t)-q_{j}(t)
$$

En revanche, si $T_{i-1}$ est validée (i.e. arrivée de véhicules) et $T_{i}$ est non validée (i.e. la sortie de véhicules est non autorisée, $\left.q_{j}(t)=0\right)$, une file d'attente se forme. La taille de cette file d'attente évolue alors selon l'équation [6] :

$$
\frac{d m_{i}(t)}{d t}=q_{j-1}(t)
$$

Les paramètres des modèles RdPCV peuvent être exprimés en fonction des paramètres du trafic sur la section de voirie $i$, à savoir le débit maximal $\phi_{\max } i$, la vitesse limitée $v_{\text {free }}$, la densité maximale $\rho_{\max i}$ (Tolba et al., 2001).

$$
\begin{gathered}
q_{\max j}=\frac{v_{\text {free } i}}{\Delta_{i}} \\
a_{j}=\frac{\phi_{\max i} \Delta_{i}}{v_{\text {free } i}} \\
c_{i}=\rho_{\max i} \cdot \Delta_{i}
\end{gathered}
$$

Dans cette contribution, on considère le trafic routier comme un ensemble de véhicules représentés par les variables moyennes de flux de circulation à savoir la densité, le débit et la vitesse. Il s'agit d'une approche macroscopique et plus particulièrement de modèles de trafic du premier ordre. L'utilisation de ces derniers a montré des limitations dans certains cas de trafic tels que, les rétrécissements des autoroutes ou les convergences avec des rampes d'accès (Papageorgiou, 1997). Pour tenter de remédier à ces défauts, le premier modèle d'ordre supérieur a été proposé en 1971 par Payne. Le modèle de Payne est présenté comme une approximation du modèle véhicule-suiveur en prenant en compte le temps de réaction du conducteur après un changement du trafic en aval, (i.e. le conducteur au point $x$ de la route ajuste sa vitesse avec un retard $\tau$ relativement à la situation du trafic au point $x+\Delta x$ avec $\Delta x>0$ ) (Papageorgiou et Schmidt, 1991).

La modélisation du trafic de façon plus fine en tenant compte de la vitesse et l'accélération individuelles de chaque véhicule ainsi qu'à la distance intervéhiculaire a été abordée dans nos travaux antérieurs (Tolba et al. 2002 ; Tolba et al. 2003 ; Tolba, 2004). Dans ces travaux nous avons modélisé une section d'autoroute par RdP discrets et comparé les résultats obtenus avec le modèle automates cellulaires du trafic (Nagel 1998).

\subsection{Modèle par RdP des intersections}

\subsubsection{Intersection isolée}

Le RdPCV présenté sur la figure 3 est bien adapté pour modéliser l'écoulement continu du trafic sur une voie du carrefour (Tolba et al., 2001). Toutefois, dans un carrefour à feux, la circulation des véhicules est inhérente aux changements d'état des feux. Dans un cycle de feu, l'évolution du trafic sur une voie est caractérisée par 
un comportement de passage pendant le vert et un comportement d'attente pendant le rouge Cette dynamique peut être modélisée par un RdPH composé de modèles élémentaires RdPCV similaires à celui de la figure 3 et d'un RdPT modélisant les feux de signalisation. La présence d'un jeton dans la place discrète $P^{*}{ }_{1}$ (représentée par un simple cercle, Figure 4) indique l'état «vert» du feu sur la voie $(O-E)$. La transition continue $T_{2}$ est donc franchissable pendant une durée du vert égale à $d_{1}$ (temporisation associée à la transition discrète $T^{*}{ }_{1}$ représentée sur la figure 4 par une barre).

En revanche, l'absence d'un jeton dans la place $P^{*}{ }_{2}$ rend la transition $T_{4}$ non franchissable (i.e., état « rouge » du feu sur la voie $(N-S)$ ). Lorsque des véhicules arrivent sur la voie $(N-S)$, ils forment une file d'attente pendant une durée égale à $d_{l}$.

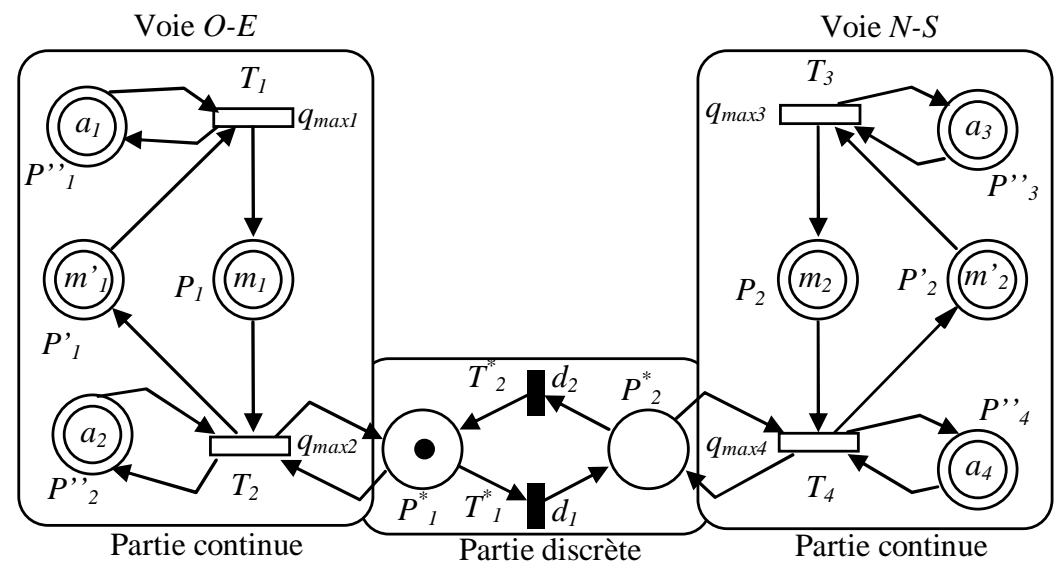

Figure 4. Modèle par RdPH du carrefour.

Pendant un cycle de feu $\left[t_{0}, t_{f}\right]$, l'écoulement du trafic dans le carrefour est régi par les deux systèmes, d'équations différentielles, suivants :

Pour $t \in\left[t_{0}, t_{c}\right]$ :

$S_{I}= \begin{cases}\dot{m}_{l}(t)=q_{\max } \min \left(a_{1}, c_{l}-m_{l}(t)\right)-q_{\max 2} \min \left(a_{2}, m_{l}(t)\right) & \text { Feu vert sur la voie } O-E \\ \dot{m}_{2}(t)=q_{\max 3} \min \left(a_{3}, c_{2}-m_{2}(t)\right) & \text { Feu rouge sur la voie } N-S\end{cases}$

avec les conditions initiales $m_{l}(0)=m_{l}{ }^{0}$ et $m_{2}(0)=m_{2}{ }^{0}$.

Pour $t \in\left[t_{c}, t_{f}\right]$ :

$S_{2}= \begin{cases}\dot{m}_{1}(t)=q_{\max } \min \left(a_{1}, c_{1}-m_{1}(t)\right) & \text { Feu rouge sur la voie } O-E \\ \dot{m}_{2}(t)=q_{\max 3} \min \left(a_{3}, c_{2}-m_{2}(t)\right)-q_{\max 4} \min \left(a_{4}, m_{2}(t)\right) & \text { Feu vert sur la voie } N-S\end{cases}$

avec les conditions initiales $m_{l}\left(t_{c}\right)$ et $m_{2}\left(t_{c}\right)$ calculées par $S_{l}$. 
Une représentation sous la forme d'un seul système qui intègre $S_{1}$ et $S_{2}$ peut être obtenue :

$S=\left\{\begin{array}{l}\dot{m}_{l}(t)=q_{\max 1} \min \left(a_{1}, c_{1}-m_{1}(t)\right)-(1-\varphi(t)) q_{\max 2} \min \left(a_{2}, m_{1}(t)\right) \\ \dot{m}_{2}(t)=q_{\max 3} \min \left(a_{3}, c_{2}-m_{2}(t)\right)-\varphi(t) q_{\max 4} \min \left(a_{4}, m_{2}(t)\right)\end{array}\right.$

où $\varphi(t)$ est une fonction discrète prenant des valeurs dans l'ensemble $\{0,1\}$ et représentant le marquage des place $P_{1}{ }^{*}$ et $P_{2}{ }^{*} . \varphi(t)$ vaut 0 quand le jeton est dans $P_{1}{ }^{*}$, il vaut 1 quand le jeton se trouve à la place $P_{2}{ }^{*}$. Dans l'exemple qui nous intéresse, puisque l'on commence le cycle par un feu vert sur la voie O-E et rouge sur la voie $\mathrm{N}-\mathrm{S}$, cela revient à prendre : $\varphi(t)=0$ pour $t \in\left[t_{0}, t_{c}\right]$ et $\varphi(t)=1$ pour $t \in\left[t_{c}, t_{f}\right]$

Les paramètres du modèle $\mathrm{RdPH}$, sont calculés en fonction des paramètres du trafic dans le carrefour [7]-[9].

\subsubsection{Réseaux d'intersections}

En se basant sur l'approche de modélisation présentée précédemment, nous allons présenter le modèle RdPH d'un réseau d'intersections interconnectées. En effet, chaque intersection est modélisée par un RdPH similaire à celui de la figure 4 . La figure 5, montre un réseau du trafic avec trois intersections $\left(I_{1}, I_{2}, I_{3}\right)$ et deux directions de circulation. On suppose que le cycle des feux de chaque intersection possède deux états « vert » et « rouge ».

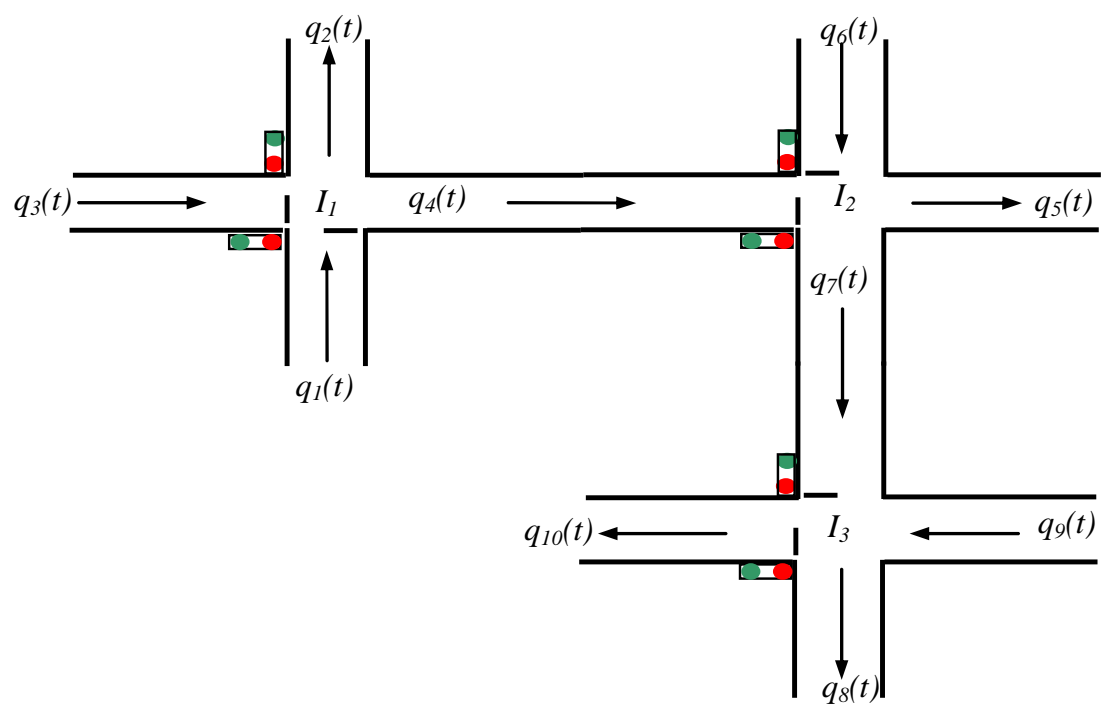

Figure 5. Description d'un réseau de trois intersections.

Le modèle par RdPH du réseau de la figure 5 peut être représenté sous la forme de trois RdPH de carrefours isolés interconnectés. 


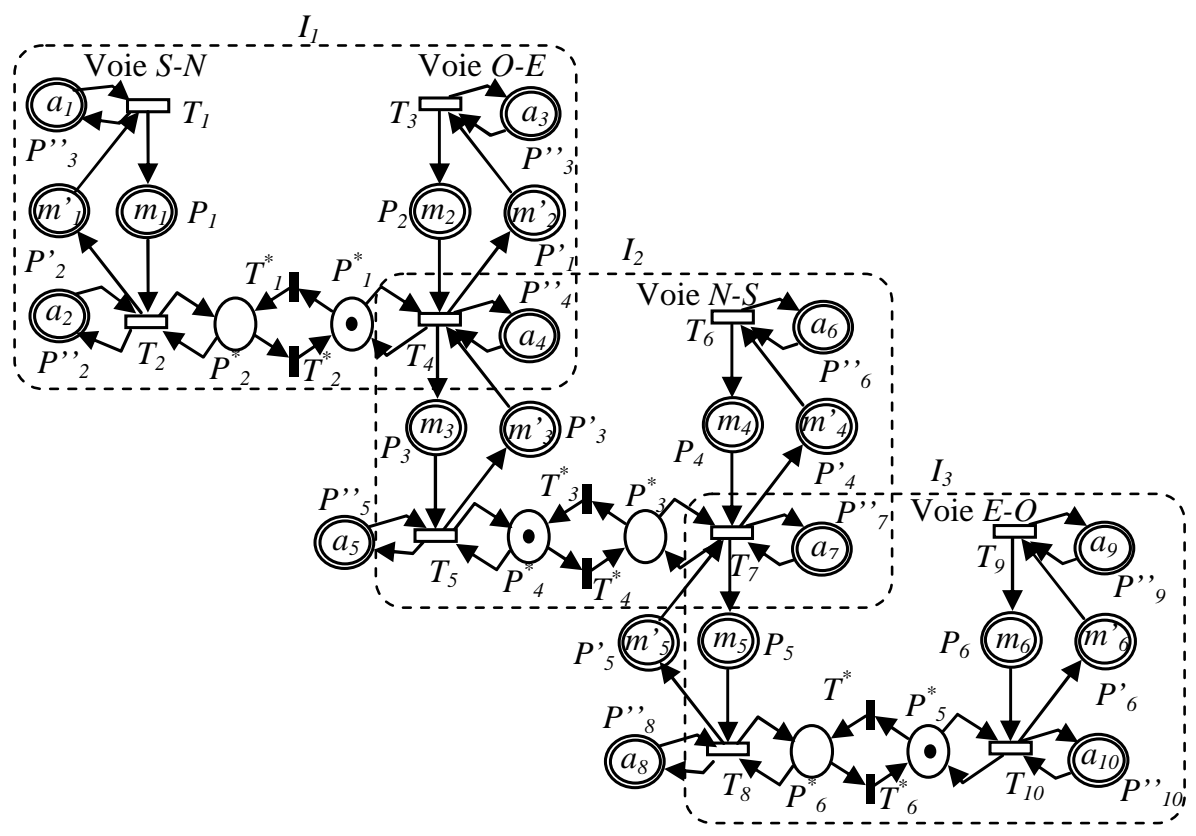

Figure 6. Modèle par RdPH de trois intersections.

En toute généralité, aucune hypothèse n'est formulée sur la synchronisation des feux. La dynamique des files d'attente pendant un cycle de feu est régie par les systèmes d'équations [13] - [15] où les fonctions $\varphi_{i}$ assurent la synchronisation, et les paramètres $q_{\max }$, $a_{i}$, et $c_{i}$ sont définis en fonction des caractéristiques du trafic:

$$
\begin{aligned}
& I_{l}=\left\{\begin{array}{l}
\dot{m}_{l}(t)=q_{\max 1} \min \left(a_{1}, c_{1}-m_{l}(t)\right)-\left(1-\varphi_{1}(t)\right) q_{\max 2} \min \left(a_{2}, m_{l}(t)\right) \\
\dot{m}_{2}(t)=q_{\max 3} \min \left(a_{3}, c_{2}-m_{2}(t)\right)-\varphi_{1}(t) q_{\max 4} \min \left(a_{4}, m_{2}(t), c_{3}-m_{3}(t)\right)
\end{array}\right. \\
& I_{2}=\left\{\begin{array}{l}
\dot{m}_{3}(t)=\varphi_{1}(t) q_{\max 4} \min \left(a_{4}, m_{2}(t), \quad c_{3}-m_{3}(t)\right)-\left(1-\varphi_{2}(t)\right) q_{\max 5} \min \left(a_{5}, m_{3}(t)\right) \\
\dot{m}_{4}(t)=q_{\max 6} \min \left(a_{6}, c_{4}-m_{4}(t)\right)-\varphi_{2}(t) q_{\max } 7 \min \left(a_{7}, m_{4}(t), \quad c_{5}-m_{5}(t)\right)
\end{array}\right. \\
& I_{3}=\left\{\begin{array}{l}
\dot{m}_{5}(t)=\varphi_{2}(t) q_{\max 7} \min \left(a_{7}, m_{4}(t), \quad c_{5}-m_{5}(t)\right)-\left(1-\varphi_{3}(t)\right) q_{\max 8} \min \left(a_{8}, m_{5}(t)\right) \\
\dot{m}_{6}(t)=q_{\max 9} \min \left(a_{9}, c_{6}-m_{6}(t)\right)-\varphi_{3}(t) q_{\max 10} \min \left(a_{10}, m_{6}(t)\right)
\end{array}\right.
\end{aligned}
$$

Si l'on considère, par exemple, que les feux de signalisation des intersections sont deux à deux synchronisés avec des déphasages constants, on peut poser $\varphi_{2}(t)=$ $\varphi_{1}\left(t+\tau_{12}\right)$ et $\varphi_{3}(t)=\varphi_{1}\left(t+\tau_{13}\right)$. 


\section{Représentation multi - modèle des intersections}

Les intersections à feux des figures 4 et 6 modélisés par des RdPH sont des systèmes hybrides non linéaires avec des commutations qui résultent de la fonction «minimum » (Tolba et al., 2004). C'est pourquoi, nous proposons une approche multi - modèle dans laquelle les systèmes d'équations [13] - [15] sont décomposés en plusieurs modèles linéaires activés séquentiellement. Chaque modèle linéaire, appelé «phase linéaire » et noté $E_{j}^{i}$, décrit l'évolution de la file d'attente $m_{i}$ par des équations différentielles linéaires du premier ordre dont l'activation dépend de la valeur des variables $m_{i}$ ainsi que des fonctions $\varphi_{i}$. De manière générale, la détermination de la phase linéaire active pendant une durée donnée est obtenue grâce à la fonction suivante (Lefebvre et al., 2004) :

$$
\begin{aligned}
& \forall T_{j}: \quad f_{j}: \quad\{1, \ldots, n\} \\
&\left(m_{i}(t)\right)_{i=1, \ldots, n} \mapsto f_{j}\left(\left(m_{i}(t)\right)_{i=1, \ldots, n}\right) \text { telle que }: m_{f_{j}}=\min \left(m_{i}(t)\right) \\
& P_{i} \in{ }^{\circ} T_{j}
\end{aligned}
$$

avec ${ }^{\circ} T_{j}$ l'ensemble des places continues $P^{\mathrm{c}}$ en amont de la transition $T_{j}$ et $f_{j}$ la fonction qui spécifie la place continue en amont de $T_{j}$ dont le marquage est minimal.

En partitionnant l'ensemble des transitions continues $T^{c}$ du RdPH modélisant le réseau de plusieurs carrefours interconnectés, il est possible de représenter la forme générale des phases linéaires. En effet, l'ensemble $T^{c}$ est divisé en 3 sous ensembles disjoints $T_{e}, T_{e s}$ et $T_{s} . T_{e}$ est l'ensemble des transitions d'entrée du réseau, $T_{e s}$ est l'ensemble des transitions de jonctions (transitions intermédiaires) et $T_{s}$ est l'ensemble des transitions de sortie du réseau. Notons $p, r$ et $s$ les dimensions de $T_{e}$, $T_{e s}$ et $T_{s}$, qui vérifient l'équation [17]:

$$
p+r+s=\operatorname{card}\left(T^{c}\right)
$$

où card(.) représente le cardinal de l'ensemble (.). Il s'ensuit que la forme générale des modèles linéaires peut être donnée par l'équation [18] :

$$
\dot{m}_{i}=\sum_{j=1}^{p} \alpha_{i j} q_{e j} m_{f_{j}}+\varphi(\cdot) \sum_{j=1}^{r} \beta_{i j} q_{e s j} m_{f_{j}}+(1-\varphi(\cdot)) \sum_{j=1}^{s} \gamma_{i j} q_{s j} m_{f_{j}}
$$

avec :

$\sum_{i=1}^{p} \alpha_{i j} q_{e j} m_{f_{i}}:$ la somme des débits d'entrée au carrefour 


$$
\begin{aligned}
& \sum_{j=1}^{r} \beta_{i j} q_{e s} m_{f_{i}}: \text { la somme des débits au point de jonction } \\
& \sum_{j=1}^{s} \gamma_{i j} q_{s j} m_{f_{i}}: \text { la somme des débits de sortie du carrefour }
\end{aligned}
$$

Les paramètres $\alpha_{i j}, \beta_{i j}$ et $\gamma_{i j}$ sont définis comme suit :

$$
\begin{gathered}
\alpha_{i j}=\operatorname{Post}\left(P_{i}, T_{e j}\right)-\operatorname{Pré}\left(P_{i}, T_{e j}\right) \\
\beta_{i j}=\operatorname{Post}\left(P_{i}, T_{e s j}\right)-\operatorname{Pré}\left(P_{i}, T_{e s j}\right) \\
\gamma_{i j}=\operatorname{Post}\left(P_{i}, T_{s j}\right)-\operatorname{Pré}\left(P_{i}, T_{s j}\right)
\end{gathered}
$$

avec Pré $: P^{c} \times T^{c} \rightarrow\{0,1\}$ la fonction de pré-incidence telle que $\operatorname{Pré}\left(P_{i}, T_{j}\right)$ est le poids de l'arc orienté de la place $P_{i}$ vers la transition $T_{j}$, et $P o s t: P^{c} \times T^{c} \rightarrow\{0,1\}$ la fonction de post-incidence telle que $\operatorname{Post}\left(P_{i}, T_{j}\right)$ est le poids de l'arc orienté de la transition $T_{j}$ vers la place $P_{i}$. Les paramètres $q_{e j}$, $q_{e s j}$ et $q_{s j}$ représentent les fréquences maximales associées respectivement aux transitions $T_{e j} \in T_{e}, T_{e s j} \in T_{e s}$ et $T_{s j} \in T_{s}$.

Pour l'exemple de la figure 5, les matrices d'incidence $\alpha, \beta, \gamma$ s'expriment en fonction des places $P_{i}$ et des transitions $T_{j}$.

$$
\alpha=\left|\begin{array}{ccccc} 
& T_{1} & T_{3} & T_{6} & T_{9} \\
P_{1} & 1 & 0 & 0 & 0 \\
P_{2} & 0 & 1 & 0 & 0 \\
P_{3} & 0 & 0 & 0 & 0 \\
P_{4} & 0 & 0 & 1 & 0 \\
P_{5} & 0 & 0 & 0 & 0 \\
P_{6} & 0 & 0 & 0 & 1
\end{array}\right| \quad \beta=\left|\begin{array}{ccc}
T_{4} & T_{7} \\
P_{1} & 0 & 0 \\
P_{2} & -1 & 0 \\
p_{3} & 1 & 0 \\
p_{4} & 0 & -1 \\
p_{5} & 0 & 1 \\
p_{6} & 0 & 0
\end{array}\right| \quad \gamma=\left|\begin{array}{ccccc} 
& T_{2} & T_{5} & T_{8} & T_{10} \\
P_{1} & -1 & 0 & 0 & 0 \\
p_{2} & 0 & 0 & 0 & 0 \\
p_{3} & 0 & -1 & 0 & 0 \\
p_{4} & 0 & 0 & 0 & 0 \\
p_{5} & 0 & 0 & -1 & 0 \\
p_{6} & 0 & 0 & 0 & -1
\end{array}\right|
$$

- $q_{e}=\left[q_{\max 1}, q_{\max 3}, q_{\max 6}, q_{\max }\right]^{\mathrm{T}}$ est associé à $T_{e}=\left[T_{1}, T_{3}, T_{6}, T_{9}\right]^{\mathrm{T}}$.

- $q_{e s}=\left[q_{\max } 4, q_{\max }\right]^{\mathrm{T}}$ est associé à $T_{e s}=\left[T_{4}, T_{7}\right]^{\mathrm{T}}$.

- $q_{s}=\left[q_{\max 2}, q_{\max 5}, q_{\max 8}, q_{\max 10}\right]^{\mathrm{T}}$ est associé à $T_{s}=\left[T_{2}, T_{5}, T_{8}, T_{10}\right]^{\mathrm{T}}$.

Les équations [13] à [15] peuvent être réécrites à l'aide des équations [20] à [22]:

$$
\begin{aligned}
& \left\{\begin{array}{l}
\dot{m}_{l}(t)=q_{\max } m_{f_{j}}(t)-\left(1-\varphi_{1}(t)\right) q_{\max 2} m_{f_{j}}(t) \\
\dot{m}_{2}(t)=q_{\max 3} m_{f_{j}}(t)-\varphi_{1}(t) q_{\max 4} m_{f_{j}}(t)
\end{array}\right. \\
& \left\{\begin{array}{l}
\dot{m}_{3}(t)=\varphi_{1}(t) q_{\max 4} m_{f_{j}}(t)-\left(1-\varphi_{2}(t)\right) q_{\max 5} m_{f_{j}}(t) \\
\dot{m}_{4}(t)=q_{\max 6} m_{f_{j}}(t)-\varphi_{2}(t) q_{\max } m_{f_{j}}(t)
\end{array}\right.
\end{aligned}
$$




$$
\left\{\begin{array}{l}
\dot{m}_{5}(t)=\varphi_{2}(t) q_{\max 7} m_{f_{j}}(t)-\left(1-\varphi_{3}(t)\right) q_{\max 8} m_{f_{j}}(t) \\
\dot{m}_{6}(t)=q_{\max 9} m_{f_{j}}(t)-\varphi_{3}(t) q_{\max 10} m_{f_{j}}(t)
\end{array}\right.
$$

Chaque phase est active pendant la durée qui sépare deux commutations successives des opérateurs «min» dans l'équation d'évolution de ces réseaux, et correspond à une configuration particulière de ces opérateurs caractérisée par les fonctions $f_{j}$. En utilisant l'équation [16], on peut remplacer les opérateurs «min » des équations [13] à [15] par les fonctions $m_{f_{j}}$ dans les équations [20] à [22].

La résolution des équations différentielles correspondant à chaque phase linéaire permet de déterminer analytiquement l'évolution du marquage mi. Le passage d'une phase linéaire à la suivante se produit lorsque le marquage mi franchit un seuil que l'on définira dans la suite, ou au moment de l'occurrence d'un événement sur la transition discrète $\mathrm{T}^{*} \mathrm{i}$ ou $\mathrm{T} *_{\mathrm{i}}+1$. Le modèle du trafic peut être représenté par un automate hybride (Tolba et al., 2004).

L'agrégation de deux automates hybrides des files d'attente, de la même intersection, mi et mi+1 permet de décrire la dynamique complète de l'intersection, Figure 5. Il s'ensuit que l'évolution globale d'un réseau de $\mathrm{N}$ carrefours peut être représenté par $\mathrm{N}$ automates hybrides interconnectés. Le réseau de la figure 6 peut être représenté par trois automates hybrides.

L'approche multi-modèles ainsi obtenue permet de mettre en relief les interactions entre la partie continue représentée par les phases linéaires et la partie discrète représentée à la fois par les changements de phases linéaires et les changements des états du feu. Par conséquent, il devient plus simple de synthétiser des commandes des feux qui reposent sur l'occurrence de ces changements que l'on appelle événements.

\subsection{Décomposition en multi-modèles linéaires}

Considérons le modèle non linéaire de l'intersection $I_{l}$ décrit par $S_{l}$ (équation [10]) et $S_{2}$ (équation [11]). Ce modèle peut être décomposé en un ensemble de phases linéaires activées séquentiellement en fonction des conditions initiales $m_{10}$ et $m_{20}$. Le modèle correspondant à chaque phase linéaire $E_{j}^{i}$ est un modèle linéaire continu décrit par une équation différentielle du premier ordre dans laquelle les fréquences de franchissement $q_{\max }, j=1,2,3$ sont considérées comme des entrées du système. Le passage d'une phase linéaire $E_{j}^{i}$ à la suivante est appelé commutation. Notons qu'une phase linéaire $E_{j}^{i}$ est différente d'une phase de feu qui correspond à la durée pendant laquelle l'état du feu ne change pas. La durée d'un cycle complet de phases d'un feu est appelée période. Chaque période comporte deux phases de feu «vert» et «rouge », et chaque phase de feu peut comporter une ou plusieurs phases linéaires. En revanche, une phase linéaire $E_{j}^{i}$ ne correspond qu'à une seule phase de feu (en changeant de phase de feu, on change aussi de phase linéaire). Une autre différence entre les phases linéaires et les phases de feu concerne la dynamique des commutations. Le changement de phase de feu correspond toujours à un événement externe (commutation des feux) alors qu'un 
changement de phase linéaire correspond soit à un événement externe, soit à un évènement interne (le marquage des places atteint un seuil). Il en résulte que l'évolution de chaque variable de marquage est donnée par six phases linéaires possibles $\left\{E_{1}^{l}, E_{2}^{l}, E^{l}, E_{4}^{l}, E^{l}{ }_{5}, E_{6}^{l}\right\}$ et $\left\{E_{1}^{2}, E_{2}^{2}, E^{2}{ }_{3}, E_{4}^{2}, E_{5}^{2}, E_{6}^{2}\right\}$. Par souci de clarté, nous présentons dans la table 1 les phases linéaires (PL) de $m_{l}$ et $m_{2}$ atteignables pendant les phases de feu «vert » et «rouge »:

\begin{tabular}{|c|c|c|}
\cline { 2 - 3 } \multicolumn{1}{c|}{} & PL atteignables pendant le vert & PL atteignables pendant le rouge \\
\hline$m_{1} / m_{2}$ & $E^{I}{ }_{1}, E_{2}^{I}, E_{3}^{I}, E_{4}^{I} / E_{1}^{2}, E_{2}^{2}, E_{3}^{2}, E_{4}^{2}$ & $E_{5}^{I}, E_{6}^{I} / E_{5}^{2}, E_{6}^{2}$ \\
\hline
\end{tabular}

Table 1. Phases linéaires de $m_{1}$ et $m_{2}$ atteignables pendant chaque phase de feu.

Chaque phase dynamique $S^{l}$ du système $S_{l}$ est représentée par une phase linéaire $E_{j}^{l}$ de $m_{l}$ validée pendant le vert et une phase linéaire $E_{j}^{2}$ de $m_{2}$ validée pendant le rouge, $S_{j}^{l}=\left(E_{j}^{l}, E_{j}^{2}\right)$. De la même façon, chaque phase dynamique $S^{2}{ }_{j}$ du système $S_{2}$ est décrite par deux phases linéaires : $E_{j}^{2}$ de $m_{2}$ validée pendant le vert et $E_{j}^{l}$ de $m_{1}$ validée pendant le rouge, $S_{j}^{2}=\left(E_{j}^{l}, E_{j}^{2}\right)$. Ceci est illustré par la figure 7 :
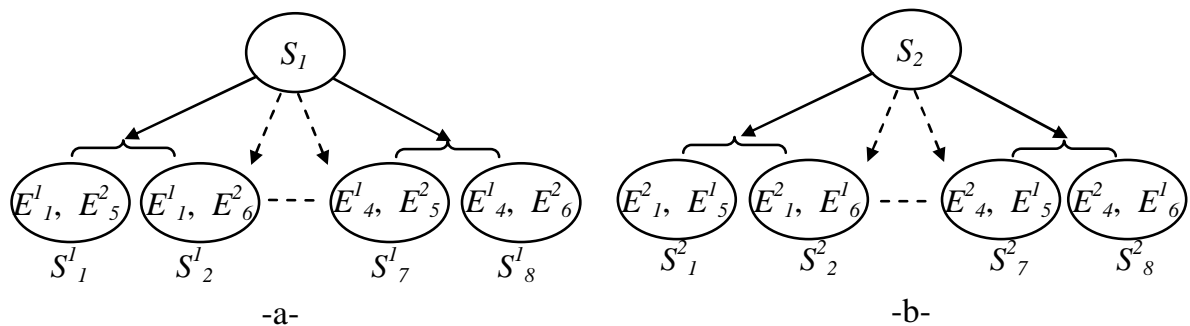

Figure 7. a-Phases linéaires de $S_{1}$, b-Phases linéaires de $S_{2}$.

La résolution des équations différentielles associées à chaque phase linéaire Eij, selon les conditions initiales, m10 et m20 et le temps initial t 0 , conduit à définir les expressions analytiques de $\mathrm{m} 1$ et $\mathrm{m} 2$. La table 2 illustre les différentes expressions de marquage associées à E1j validées dans leurs domaines respectifs. Supposons que a2<c1-a1, il en résulte que la phase linéaire E14, représentée par une case sur fond gris dans la table 2, n'est jamais validée. 
C. Tolba, D. Lefebvre, P. Thomas, A. ElMoudni, « Commande des feux de signalisation par réseaux de Petri hybrides », JESA, Vol.42, n5, pp 579-612, 2008.

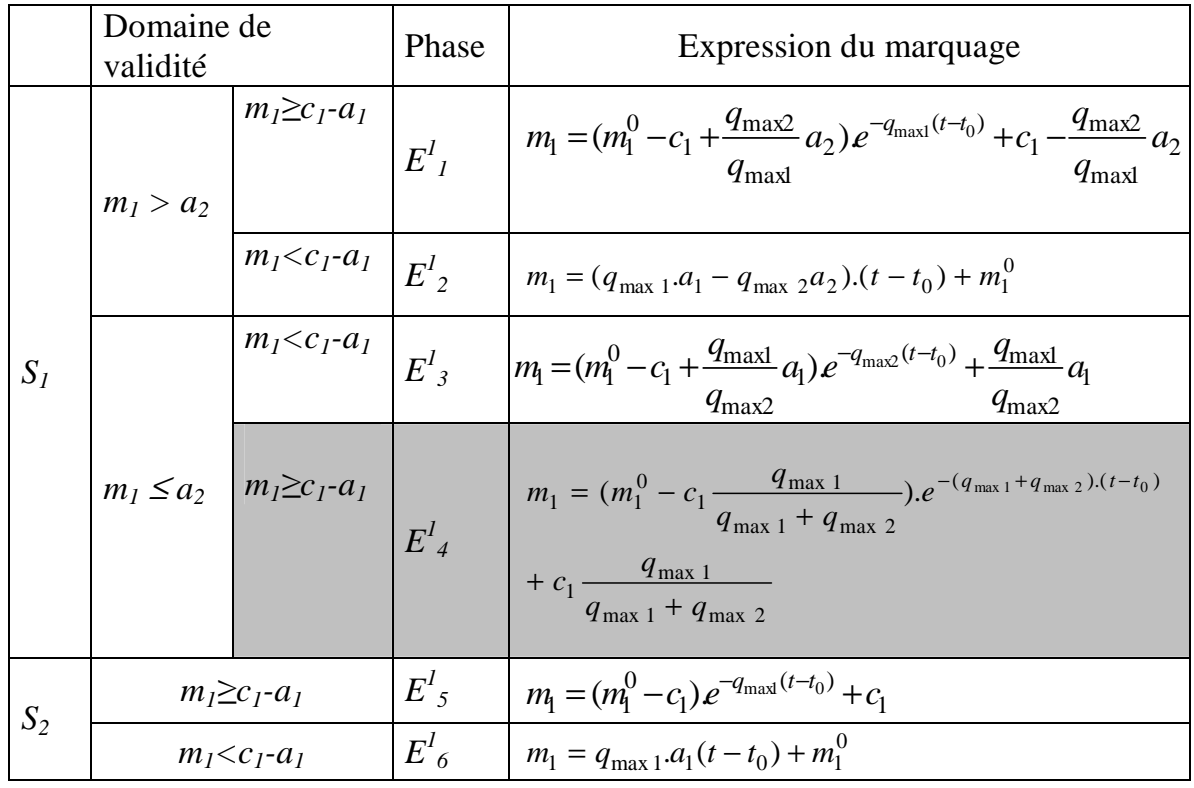

Table 2. Phases linéaires pour $m_{l}$.

De manière similaire à $m_{1}$, il est possible de définir les expressions de marquage $m_{2}$ associées à chaque phase linéaire $E_{j}^{2}$.

\subsection{Commutation des modèles linéaires}

Si l'on s'intéresse uniquement au marquage $m_{l}$, le changement de phases $E_{j}^{l}$, $j=1, \ldots, 6$ est obtenu par commutations selon l'automate ci-dessous, Figure 8 -a. Les transitions en gras correspondent à des changements de phase de feu agissant sur la partie discrète du modèle par RdP hybride $\boldsymbol{E} \boldsymbol{v}=\boldsymbol{T}^{*}{ }_{1}, \boldsymbol{T}^{*}{ }_{2}$. Les autres événements correspondent à des ruptures de seuils de la partie continue. Un automate similaire existe aussi pour $m_{2}$, Figure 8-b. 


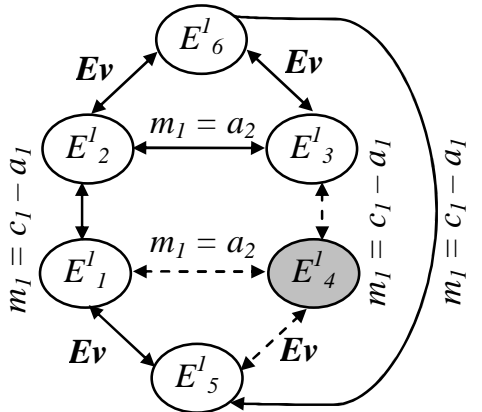

$-a-$

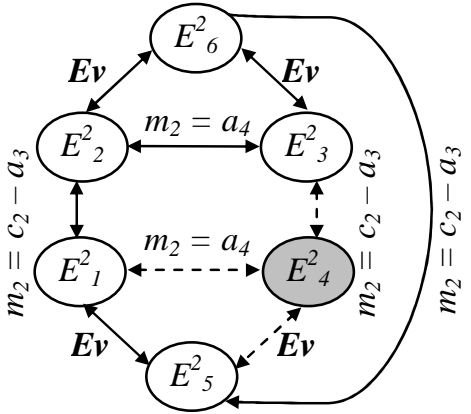

$-b-$

Les événements conduisant aux phases linéaires $E_{4}^{l}$ et $E^{2}{ }_{4}$ sont représentés par des arcs discontinus.

Figure 8. Commutation de phases linéaires a) pour $m_{1}$, b) pour $m_{2}$.

La synchronisation des deux automates via les changements de phases de feu et une horloge commune permettent d'accéder aux différentes phases linéaires. Le modèle complet comporte 16 phases linéaires différentes, Table 3. Le passage d'une phase linéaire à l'autre est commandé par l'évolution de l'automate, Figure 9, résultant de la synchronisation des deux automates de la figure 8.

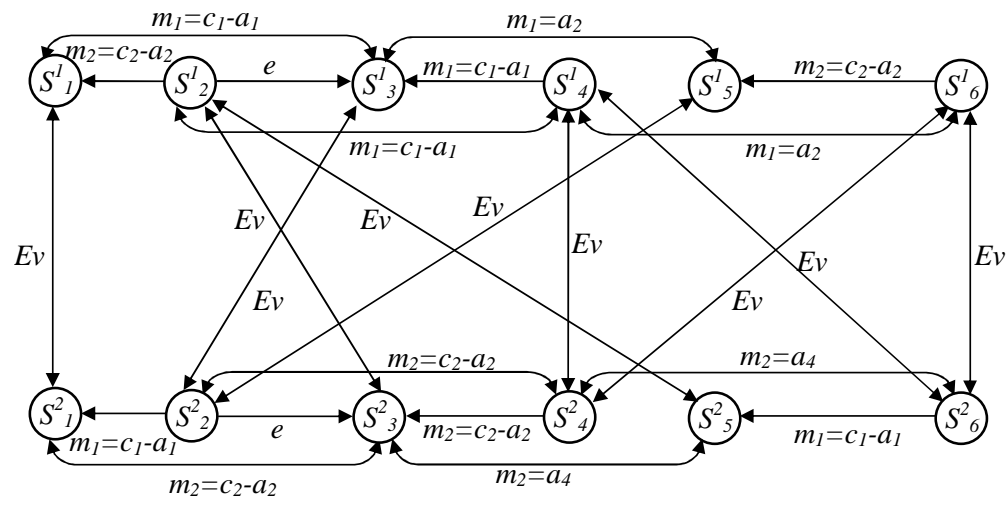

Occurrence d'un événement sur la transition $T_{1}^{*}$ ou $T_{2}^{*}: \boldsymbol{E} \boldsymbol{v}=\boldsymbol{T}_{1}^{*}, \boldsymbol{T}_{2}^{*}$ $e=\left\langle m_{1}=c_{1}-a_{1}\right.$ et $m_{2}=c_{2}-a_{2} »$

Figure 9. Automate de synchronisation 


\begin{tabular}{|c|c|c|c|c|c|c|c|c|c|}
\hline \multirow{4}{*}{\multicolumn{4}{|c|}{$\begin{array}{c}\text { Phases linéaires pour } m_{1} \\
\text { et } m_{2}\end{array}$}} & \multicolumn{4}{|c|}{$S_{1}$} & \multirow{2}{*}{\multicolumn{2}{|c|}{$S_{2}$}} \\
\hline & & & & \multicolumn{2}{|c|}{$m_{1}>a_{2}$} & \multicolumn{2}{|c|}{$m_{1} \leq a_{2}$} & & \\
\hline & & & & \multirow{2}{*}{\begin{tabular}{|c|}
$m_{1} \geq c_{1}-a_{l}$ \\
$\boldsymbol{E}^{I}$
\end{tabular}} & \multirow{2}{*}{$\frac{m_{1}<c_{1}-a_{1}}{\boldsymbol{E}^{I}{ }_{2}}$} & \multirow{2}{*}{$\frac{m_{1}<c_{1}-a_{l}}{\boldsymbol{E}_{3}^{I}}$} & $m_{1} \geq c_{1}-a_{l}$ & \multirow{2}{*}{$\frac{m_{1} \geq c_{1}-a_{1}}{\boldsymbol{E}^{I}{ }_{5}}$} & \multirow{2}{*}{$\frac{m_{1}<c_{1}-a_{1}}{\boldsymbol{E}_{6}^{I}}$} \\
\hline & & & & & & & $E_{4}^{I}$ & & \\
\hline \multirow[b]{2}{*}{$S_{1}$} & $m_{2}$ & $\geq c_{2}-a_{3}$ & $E^{2}{ }_{5}$ & $S_{1}^{1}$ & $S_{3}^{1}$ & $S_{5}^{I}$ & $S_{7}^{I}$ & $X$ & $\mathrm{X}$ \\
\hline & $m_{2}$ & $c_{2}-a_{3}$ & $E_{6}^{2}$ & $S_{2}^{I}$ & $S_{4}^{I}$ & $S_{6}^{1}$ & $S_{8}^{1}$ & $\mathrm{X}$ & $\mathrm{X}$ \\
\hline \multirow{4}{*}{$S_{2}$} & $m_{2}>a_{4}$ & $m_{2} \geq c_{2}-a_{3}$ & $E_{1}^{2}$ & $X$ & $X$ & $X$ & $X$ & $S_{1}^{2}$ & $S_{2}^{2}$ \\
\hline & & $m_{2}<c_{2}-a_{3}$ & $E_{2}^{2}$ & $X$ & $X$ & $X$ & $X$ & $S_{3}^{2}$ & $S_{4}^{2}$ \\
\hline & $m_{2} \leq a_{4}$ & $m_{2}<c_{2}-a_{3}$ & $E_{3}^{2}$ & $\mathrm{X}$ & $\mathrm{X}$ & $\bar{X}$ & $\mathrm{X}$ & $S^{2}{ }_{5}$ & $S_{6}^{2}$ \\
\hline & & $m_{2} \geq c_{2}-a_{3}$ & $E_{4}^{2}$ & $X$ & $\bar{X}$ & $\bar{X}$ & $X$ & $S_{7}^{2}$ & $S_{8}^{2}$ \\
\hline
\end{tabular}

Table 3. Phases linéaires pour le modèle $S_{1}$ et $S_{2}$.

\section{Commande événementielle locale des feux de signalisation}

Sur les figures 8 et 9 , nous pouvons remarquer que le franchissement des seuils caractérise la situation du trafic et permet ainsi de délivrer ou d'inhiber l'ordre de commutation du feu. Dans cette étude nous allons exploiter les seuils naturels du multi - modèles pour la commande du feu. Le déclenchement de l'ordre de commutation de $S_{1}$ à $S_{2}$ et vice versa $\left(T^{*}, T_{2}{ }_{2}\right)$ est délivré lorsque le marquage de la file d'attente atteint l'un des seuils $m_{i}=a_{j}$, ou $m_{i}=c_{j}$ - $a_{j}$ après un temps au moins égal à un premier seuil $G_{m}$ appelé «vert minimum » et ne dépassant pas un second seuil $G_{M}$ appelé « vert maximum ».

\subsection{Stratégies de commande}

Dans cette section nous allons présenter trois stratégies de commande des feux de signalisation en se basant sur la détection de la rupture de seuils naturels.

\subsubsection{Politique de maintien (seuils bas)}

Cette stratégie consiste à détecter la rupture des seuils bas $\left(m_{1}=a_{2}\right.$ et $\left.m_{2}=a_{4}\right)$, sur la voie dont la phase de feu est « vert », quel que soit l'état de la file d'attente sur la voie dont la phase de feu est « rouge ». Il s'agit de privilégier la résorption de la file d'attente sur la voie dont la phase de feu est «vert ». En conséquence les automates hybrides de la figure 8 et 9 se simplifient, Figure 10 et 11 .

Si l'on considère que la phase de feu est « vert » sur la voie $(O-E)$, alors l'évolution du marquage $m_{l}$ peut être exprimée par les phases linéaires $E_{l}^{l}, E_{2}^{l}$ et $E_{3}^{l}$ (rappelons que $E^{l}{ }_{4}$ n'est jamais validée). Supposons maintenant qu'à l'état initial la phase linéaire $E_{2}^{l}$ est validée, alors l'évolution de $m_{l}$ va durer tant que $\left(m_{l}>a_{2}\right.$ et $\left.m_{1}<c_{1}-a_{l}\right)$. Lorsque le seuil $m_{l}=a_{2}$ est franchi et la durée de la phase de feu « vert » $d_{\text {feu }}$ est au moins égale à $G_{m}$, l'ordre de commutation du feu est délivré. Par conséquent, le marquage $m_{l}$ change de phase linéaire (i.e., le marquage passe de $E_{2}^{l}$ 
à $E_{6}^{l}$, cette transition est représentée par un arc discontinu sur la figure 10 . En revanche, lorsque le seuil $m_{l}=a_{2}$ est franchi après une durée inférieure à $G_{m}$, le marquage $m_{l}$ change de phase linéaire et atteint $E_{3}^{l}$. A partir de cette phase linéaire, l'ordre de commutation des feux est susceptible d'être délivré lorsque $d_{f e u}=G_{m}$. Dans le cas où le marquage $m_{1}$ atteint la phase linéaire $E_{1}^{l}$ et le seuil $m_{l}>a_{2}$ n'est pas atteint, l'ordre de commutation des feux doit être donné lorsque $d_{f e u}=G_{M}$.

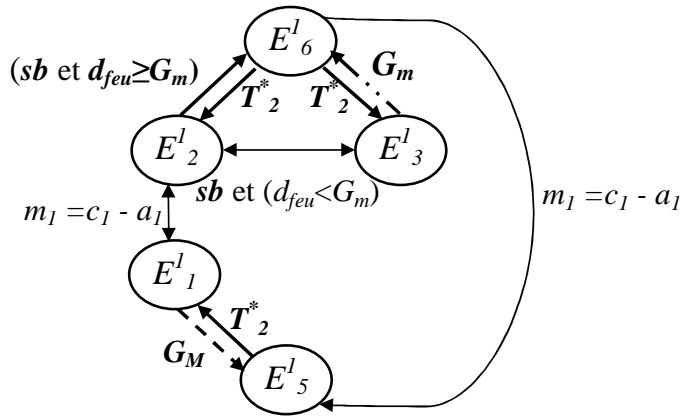

Evènement seuil bas : $s b=\ll m_{l}=a_{2} »$.

Figure 10. Automate simplifié de $m_{1}$.

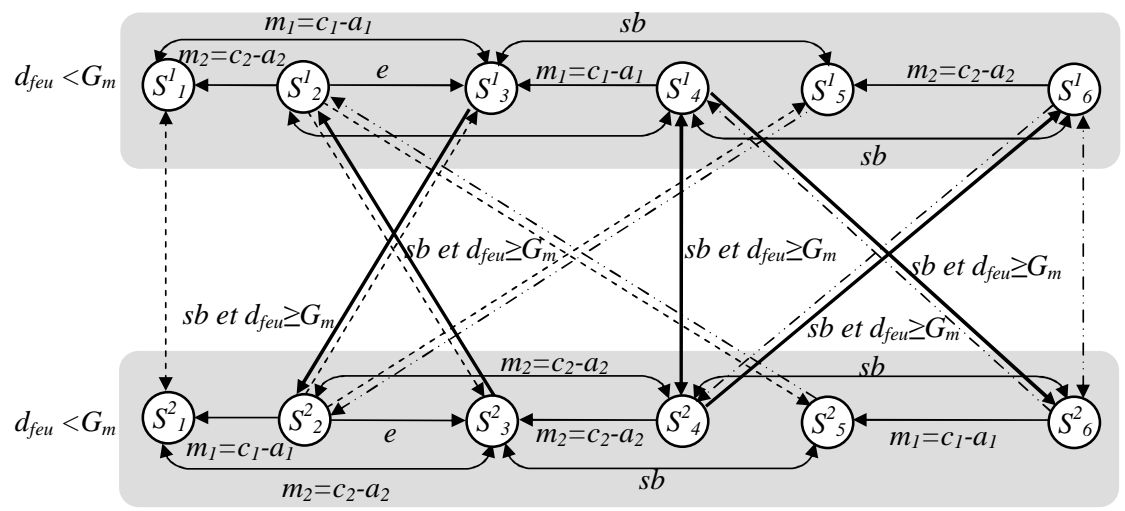

Rupture du seuil bas $s b=\ll m_{1}=a_{2} »$ ou « $m_{2}=a_{4} »$

$\rightarrow$ arc étiqueté par l'évènement : $d_{f e u} \geq G_{m}$

$e=\left\langle m_{1}=c_{1}-a_{1}\right.$ et $m_{2}=c_{2}-a_{2} »$

$\rightarrow$ arc étiqueté par l'évènement : $\mathrm{d}_{f e u} \geq G_{M}$

Figure 11. Simplification de l'automate de synchronisation. 


\subsubsection{Politique de commutation (seuils hauts)}

Contrairement à la commande précédente, il s'agit cette fois de la détection de la rupture des seuils hauts $m_{1}=c_{1}-a_{1}$, ou $m_{2}=c_{2}-a_{3}$ sur la voie dont l'état du feu est « rouge », quel que soit l'état de la file d'attente sur la voie dont la phase de feu est « vert ». Il s'agit d'empêcher la formation de la file d'attente sur la voie dont la phase de feu est " rouge ». Lorsque le marquage de la file d'attente au feu rouge atteint le seuil haut et $d_{f e u} \geq G_{m}$, le feu change de phase et par conséquent le marquage correspondant à cette file d'attente change de phase linéaire. L'automate hybride de la figure 12 illustre le fonctionnement de cet algorithme de commande.

Notons que l'évolution du marquage $m_{2}$ conformément à cet algorithme peut être représentée par un automate similaire à celui de la figure 12 .

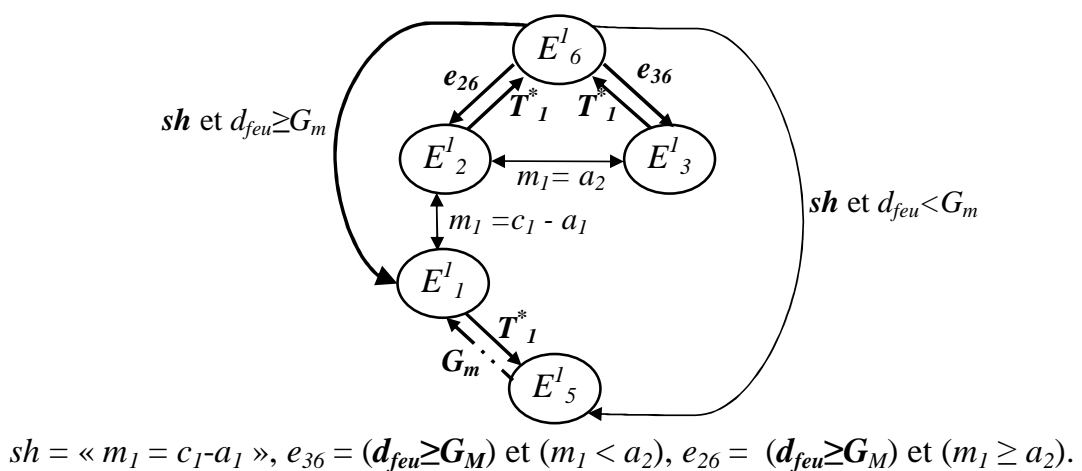

Figure 12. Automate simplifié de $m_{1}$.

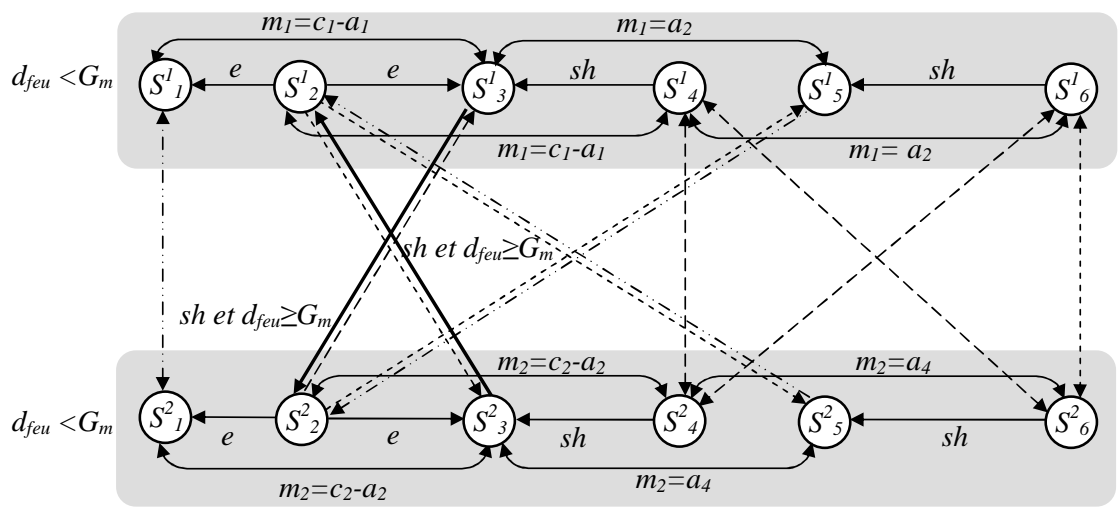

$s h=\left\langle m_{1}=c_{1}-a_{1} »\right.$ ou $\left\langle m_{2}=c_{2}-a_{2} » ; e=\left\langle m_{1}=c_{1}-a_{1}\right.\right.$ et $m_{2}=c_{2}-a_{2} »$

$\rightarrow \rightarrow$ arc étiqueté par l'évènement $: d_{\text {feu }} \geq G_{m} \rightarrow$ arc étiqueté par l'évènement $: \mathrm{d}_{\text {feu }} \geq G_{M}$

Figure 13. Simplification de l'automate de synchronisation. 


\subsubsection{Commande mixte (seuils bas et hauts)}

Cette commande repose sur la détection des seuils bas et hauts sur les deux voies de l'intersection. Il s'agit à la fois d'empêcher la formation de la file d'attente sur la voie dont la phase de feu est « rouge » et de résorber la file d'attente sur la voie dont la phase de feu est «vert». Les feux peuvent changer de phase soit lors du franchissement des seuils bas $\left(m_{1}=a_{2}, m_{2}=a_{4}\right)$, soit lors du franchissement des seuils hauts $\left(m_{1}=c_{1}-a_{1}, m_{2}=c_{2}-a_{3}\right)$. Lorsque l'un des seuils est détecté, après une durée de vert au moins égale à $G_{m}$, les feux changent d'états. L'automate de la figure 14 illustre la dynamique de $m_{1}$ conformément à cet algorithme de commande. Il existe un automate similaire pour $m_{2}$.

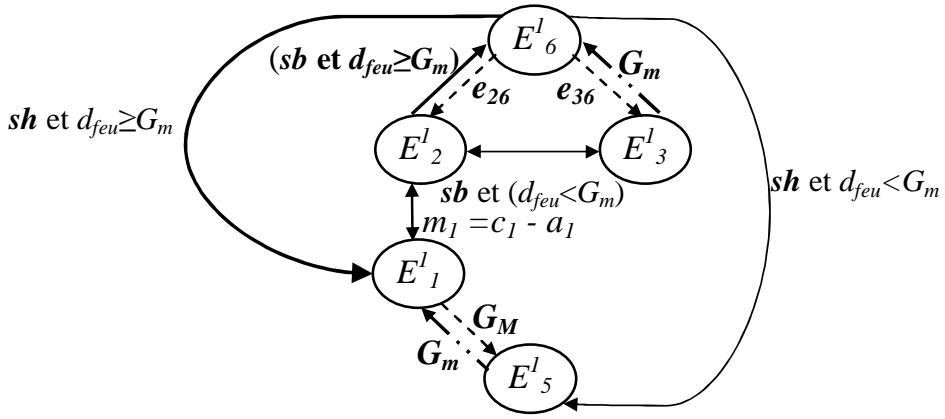

Figure 14. Automate simplifié de $m_{l}$.

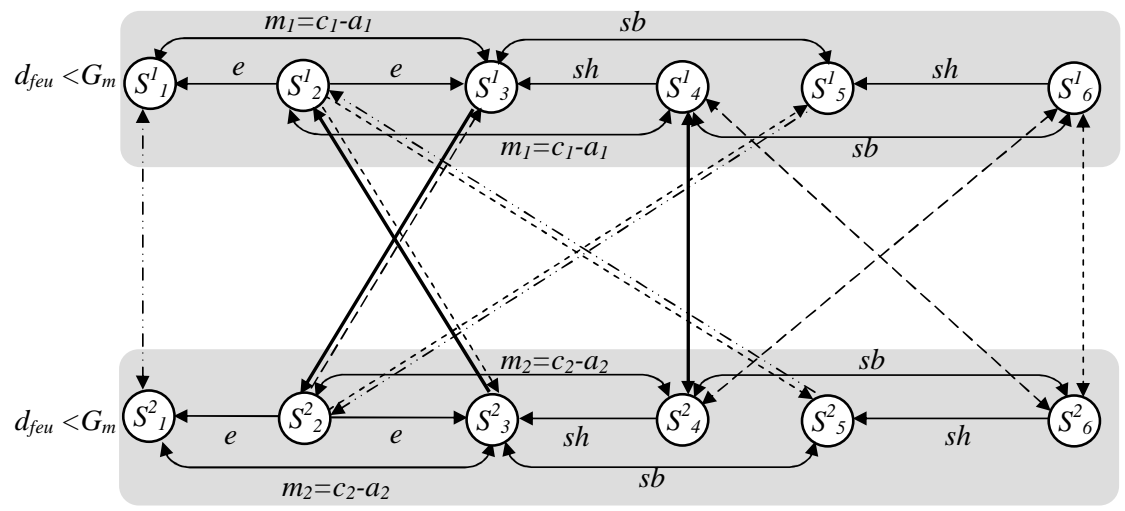

$s h=\ll m_{1}=c_{1}-a_{1} »$ ou $« m_{2}=c_{2}-a_{2} » ; e=\ll m_{1}=c_{1}-a_{1}$ et $m_{2}=c_{2}-a_{2} »$

$\longrightarrow$ arc étiqueté par l'évènement : $(s b$ ou $s h)$ et $d_{f e u} \geq G_{m}$

$\rightarrow \rightarrow$ arc étiqueté par l'évènement $: d_{f e u} \geq G_{m} ;-\cdots$ arc étiqueté par l'évènement $: \mathrm{d}_{f e u} \geq G_{M}$

Figure 15. Simplification de l'automate de synchronisation. 


\subsection{Algorithme de commande}

Les trois algorithmes de commande résultant se résument comme suit :

Etape $1:$ Initialiser $m_{1}\left(t_{0}\right), m_{2}\left(t_{0}\right)$ et $d_{\text {feu }}$

Etape 2 : Evaluer la durée d'activation de la phase linéaire $d_{l i n}$

Etape 3 : Evaluer la durée d'activation de la phase du feu $d_{f e u}$

Etape 4 : Test sur $d_{f e u}$

4.1. Si $d_{f e u}<G_{m}$

4.1.2. Evaluer les longueurs des files d'attente $m_{1}$ et $m_{2}$

4.1.3. Aller à l'étape 2

4.2. Si $G_{m} \leq d_{f e u}<G_{M}$

4.2.1. Evaluer les longueurs des files d'attente $m_{1}$ et $m_{2}$

4.2.2. Appliquer la règle de commutation des feux adéquate, Table 4

4.2.3. Sinon aller à l'étape 2

4.3. $\operatorname{Si} d_{\text {feu }} \geq G_{M}$

4.3.1. $d_{f e u}=d_{f e u}-d_{l i n}$

4.3.2. $d_{\text {lin }}=G_{M}-d_{f e u}$.

4.3.3. Evaluer les longueurs des files d'attente $m_{1}$ et $m_{2}$

Etape 5 : Fin

4.3.4. Commuter le feu et aller à l'étape 1

\begin{tabular}{|c|l|l|}
\hline $\begin{array}{c}\text { Type de } \\
\text { commande }\end{array}$ & $\begin{array}{l}\text { Voie de détection } \\
\text { de véhicule }\end{array}$ & \multicolumn{1}{|c|}{ de l'algorithme de commande } \\
\hline $\begin{array}{c}\text { Politique de } \\
\text { maintien }\end{array}$ & $\begin{array}{l}\text { File d'attente au } \\
\text { feu vert }\end{array}$ & $\begin{array}{l}\text { Si } m_{i} \leq a_{j}(i=1,2 \text { et } j=2,4) \\
\text { Commuter les feux et aller à l'étape 1. }\end{array}$ \\
\hline $\begin{array}{c}\text { Politique de } \\
\text { commutation }\end{array}$ & $\begin{array}{l}\text { File d'attente au } \\
\text { feu rouge }\end{array}$ & $\begin{array}{l}\mathrm{Si} c_{i^{-}} m_{i} \leq a_{j}(i=1,2 \text { et } j=1,3) \\
\text { Commuter les feux et aller à l'étape 1. }\end{array}$ \\
\hline $\begin{array}{c}\text { Commande } \\
\text { mixte }\end{array}$ & $\begin{array}{l}\text { Files d'attente sur } \\
\text { les deux voies }\end{array}$ & $\begin{array}{l}\mathrm{Si} m_{i} \leq a_{j}(i=1,2 \text { et } j=2,4) \text { (file d'attente } \\
\text { au feu vert) } \\
\text { Ou } \\
\text { Si } c_{i^{-}} m_{i} \leq a_{j}(i=1,2 \text { et } j=1,3) \text { (file d'attente } \\
\text { au feu rouge }) \\
\text { Commuter les feux et aller à l'étape 1. }\end{array}$ \\
\hline
\end{tabular}

Table 4. Règles de commutation des feux.

\section{Simulations}

Dans cette section, nous allons simuler l'évolution du trafic dans un carrefour isolé dans lequel les feux de signalisation commutent sur l'occurrence de la rupture 
de seuils bas et hauts. Les performances de cette commande seront comparées à celles des commandes usuelles (cycle fixe et intervalle-véhicule). Ensuite nous allons élargir l'application de cet algorithme au réseau d'intersections de la figure 5.

\subsection{Commande à cycle fixe}

Le changement de phases de feu est obtenu en fonction d'un échéancier, (i.e. la durée du cycle est prédéterminée). Dans ce cas l'occurrence des franchissements des transitions $T_{1}^{*}$ et $T_{2}^{*}$ dépend uniquement de l'horloge commune. Dans les simulations qui suivent l'horizon est de 300 secondes et le feu est supposé à cycle fixe de durée égale à 2 minutes.

\subsection{Commande intervalle - véhicule}

Cette stratégie est la plus utilisée (Papageorgiou et al., 2003). Au début de la phase, on donne au temps de vert une durée minimale $G_{m}$. Si aucun véhicule n'a été détecté pendant ce temps, le vert est alors coupé et la phase suivante commence. Le même processus est ensuite appliqué sur la seconde voie de circulation. Si au contraire, le détecteur est informé du passage d'un véhicule, la durée du vert est prolongée d'un temps $\Delta t$. Cette prolongation est fixe, elle doit permettre au véhicule de franchir la distance entre la boucle et la ligne d'arrêt. Une nouvelle prolongation du vert est accordée si un nouveau véhicule se présente pendant un intervalle de temps $I$ appelé intervalle véhiculaire. Cet intervalle est l'écart maximum entre deux véhicules au-delà duquel aucune prolongation n'est accordée au suivant. Pendant chaque intervalle véhiculaire on observe si un nouveau véhicule est détecté afin d'accorder une nouvelle prolongation de la durée du vert. Dans le cas où aucun véhicule n'est détecté le feu passe à la phase suivante. Afin d'éviter une conservation du vert sur une durée trop longue sa prolongation est contrainte par une durée maximale, appelée vert maximum qui ne pourra être dépassée quelle que soit la demande pour la phase considérée.

\subsection{Commande d'un carrefour isolé}

Dans cette série de simulations, nous allons considérer que les arrivées de véhicules suivent une loi exponentielle de paramètre $\mu$. Il s'ensuit donc que la probabilité que le délai $h$ entre deux arrivées successives de véhicules, soit supérieur ou égal à $t$, est donnée par [23]:

$$
\operatorname{Pr}(h \geq t)=\mu e^{-\mu t}
$$

où $T=1 / \mu$ représente le délai moyen entre deux arrivées successives de véhicules.

Bien que cette distribution se révèle adaptée à l'approximation des arrivées de véhicules, elle fournit parfois des données irréalistes lorsque $h$ est plus petit que l'intervalle véhiculaire. Afin de permettre une utilisation plus réaliste de ces 
données, nous introduisons une durée minimale $\left(T_{\min }\right)$ garantissant l'écart de temps minimal entre deux arrivées successives de véhicules.

Considérons le carrefour isolé présenté précédemment, Figure 1. Nous allons appliquer aux feux de ce carrefour l'algorithme de commande mixte ainsi que les commandes usuelles. Cette série de simulations est effectuée avec les paramètres suivants :

\begin{tabular}{|c|c|c|c|c|}
\hline \multicolumn{2}{|c|}{ Carrefour } & \multicolumn{2}{|c|}{$\mathrm{RdPH}$} & Commandes \\
\hline Vitesse libre & $54 \mathrm{~km} / \mathrm{h}$ & $q_{\max j}$ & $0,5 \mathrm{hz}$ & $I=4 \mathrm{~s}$ \\
\hline Longueur de la voie & $40 \mathrm{~m}$ & $a_{j}$ & 1 véh. & $G_{m}=10 \mathrm{~s}$ \\
\hline Capacité de la voie & 8 véh. & $c_{i}$ & 8 véh. & $G_{M}=60 \mathrm{~s}$ \\
\hline Débit maximal de sortie & 1 véh/s & & & $T_{\min }=2 \mathrm{~s}$ \\
\hline
\end{tabular}

Table 5. Paramètres d'un carrefour isolé, du modèle par RdPH et de la commande.
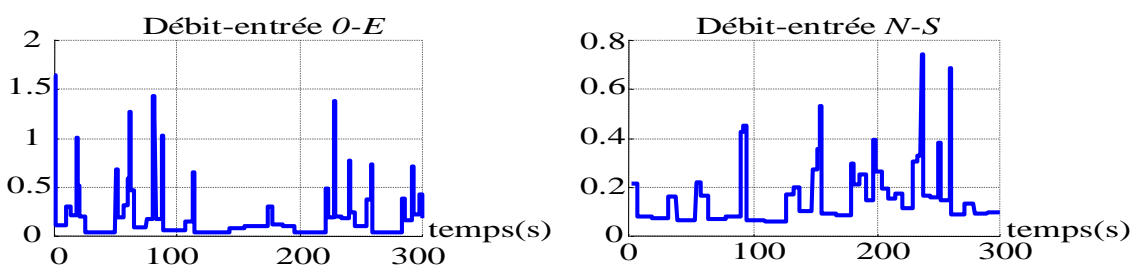

Figure 16. Profils des arrivées de véhicules.

La figure 16 présente les intervalles de temps séparant l'arrivée de deux véhicules successifs sur les voies $(O-E)$ et $(N-S)$. On peut alors constater que dans l'intervalle $[0,129 \mathrm{~s}]$, le trafic est caractérisé par un faible flux d'arrivée de véhicules sur la voie $(N-S)$ comparativement à la voie $(O-E)$. 
C. Tolba, D. Lefebvre, P. Thomas, A. ElMoudni, « Commande des feux de signalisation par réseaux de Petri hybrides », JESA, Vol.42, n5, pp 579-612, 2008.
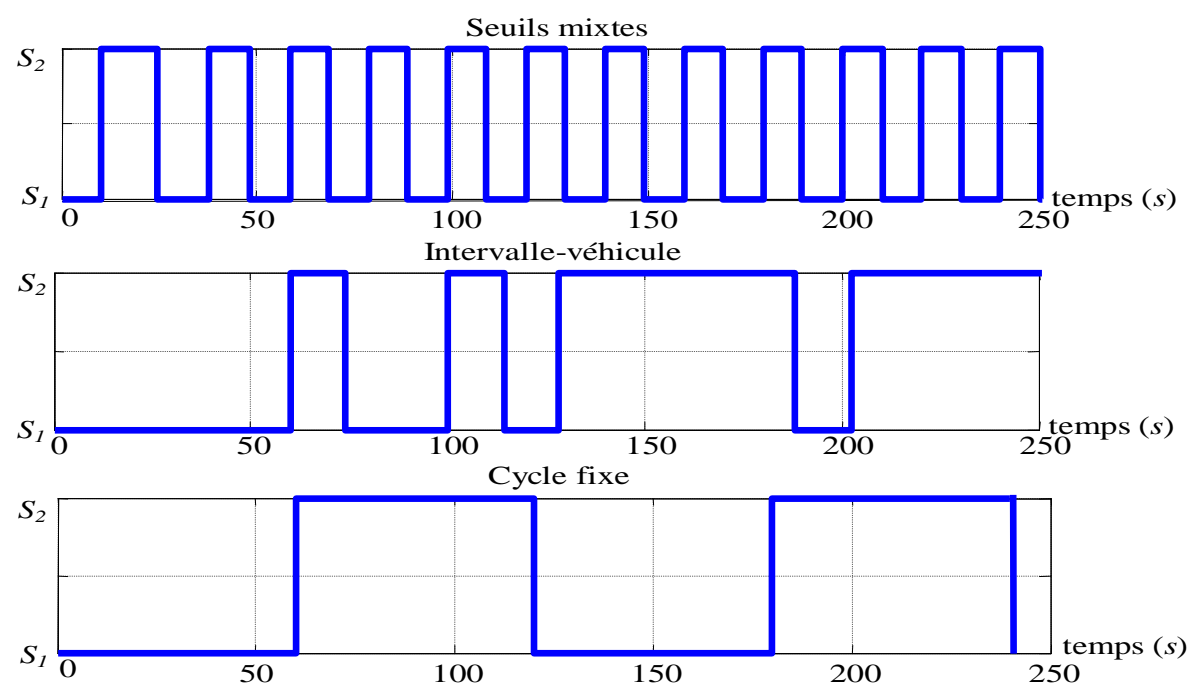

Figure 17. Séquences de commutation des feux.
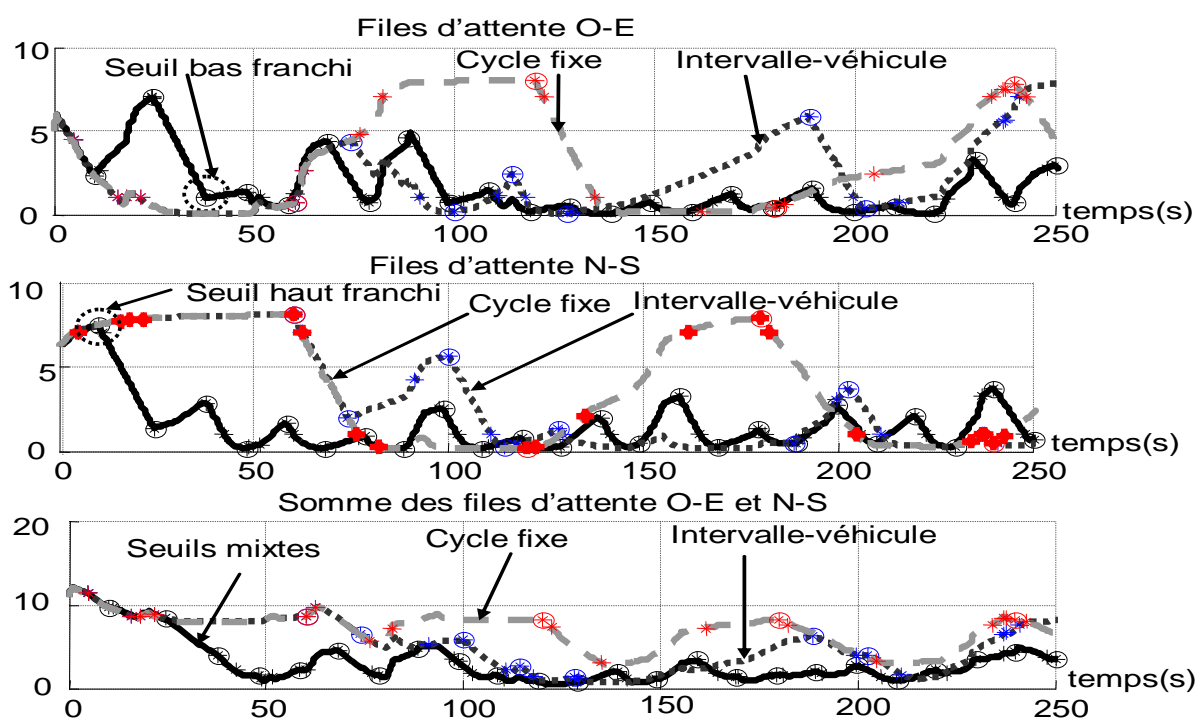

Figure 18. Evolution des files d'attente.

Les instants de commutation des feux sont présentés par la figure 17 pour les trois commandes différentes. $S 1$ indique que les véhicules circulant sur la voie $(O-E)$ 
ont le feu vert (rouge pour $(N-S)$ ) quand $S 2$ indique que ce sont ceux, circulant sur la voie $(N-S)$, qui ont le feu vert (rouge pour $(O-E)$ ). La figure 18 présente l'évolution des files d'attente pour les trois commandes (cycle fixe : tiret gris clair ; intervalle véhicule : pointillé gris foncé ; seuils mixtes : noir continu). Cette figure montre que dès l'instant $25 \mathrm{~s}$, la commande à rupture de seuils mixtes fournit de meilleurs résultats que ses deux concurrentes. En particulier, durant l'intervalle de temps [25s, $110 \mathrm{~s}$ ], cette commande est la seule à prendre en compte correctement le flux d'arrivé de véhicules sur la voie $(N-S)$.

Le rapport $\frac{\text { file d'attente pendant le rouge }}{\text { capacité maximale du carrefour }}$ (figure 19) confirme que la commande à rupture de seuils est plus performante que ses deux concurrentes et permet de minimiser le temps d'attente d'un véhicule au carrefour.

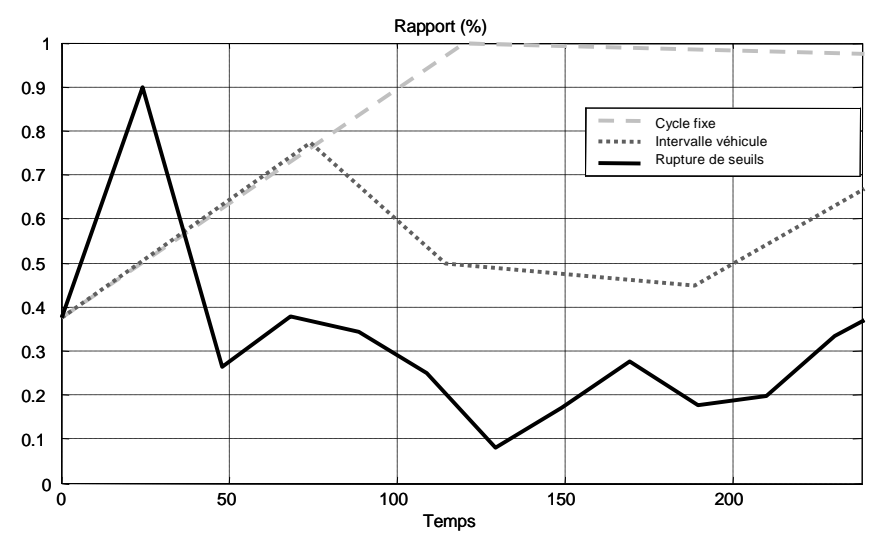

Figure 19. Ratio files d'attente / capacité pendant le rouge.
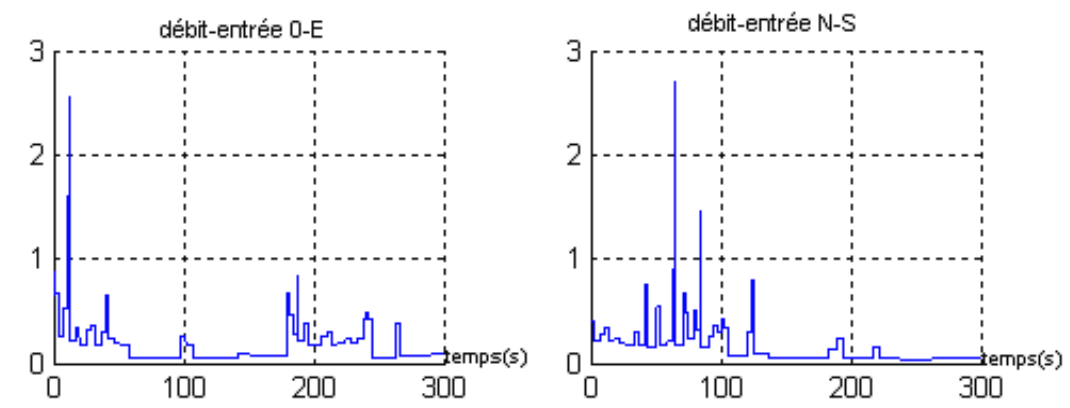

Figure 20. Profils des arrivées de véhicules. 
Afin de montrer que ces résultats ne sont pas tributaires du jeu de donnée, La figure 20 présente les intervalles de temps séparant l'arrivée de deux véhicules successifs sur les voies $(O-E)$ et $(N-S)$ pour un autre exemple. Le trafic est cette fois caractérisé par des flux similaires sur les deux voies de trafic jusqu'à l'instant $120 \mathrm{~s}$, instant où il devient beaucoup plus dense sur la voie $(N-S)$.
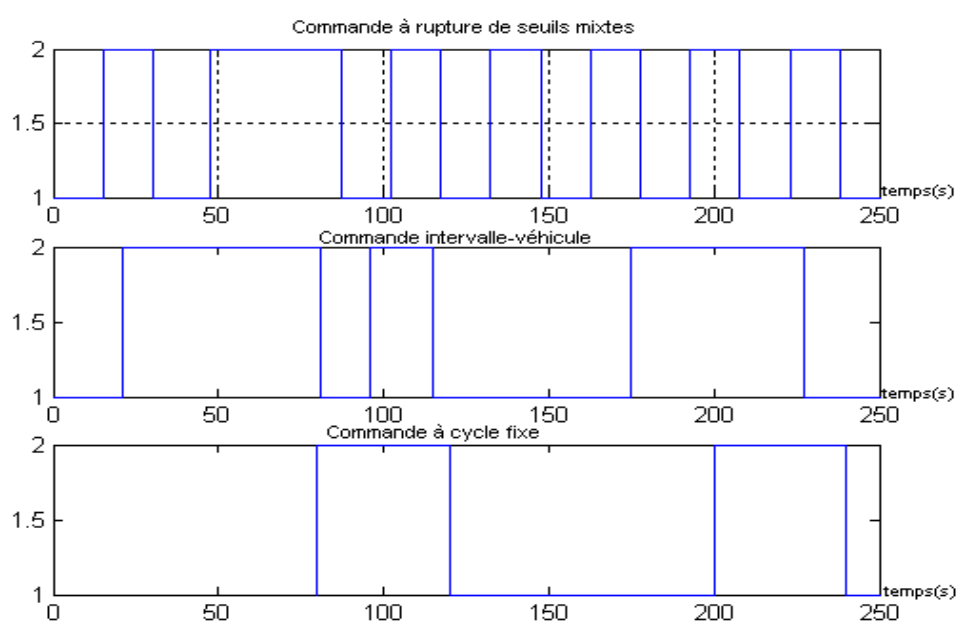

Figure 21. Séquences de commutation des feux.
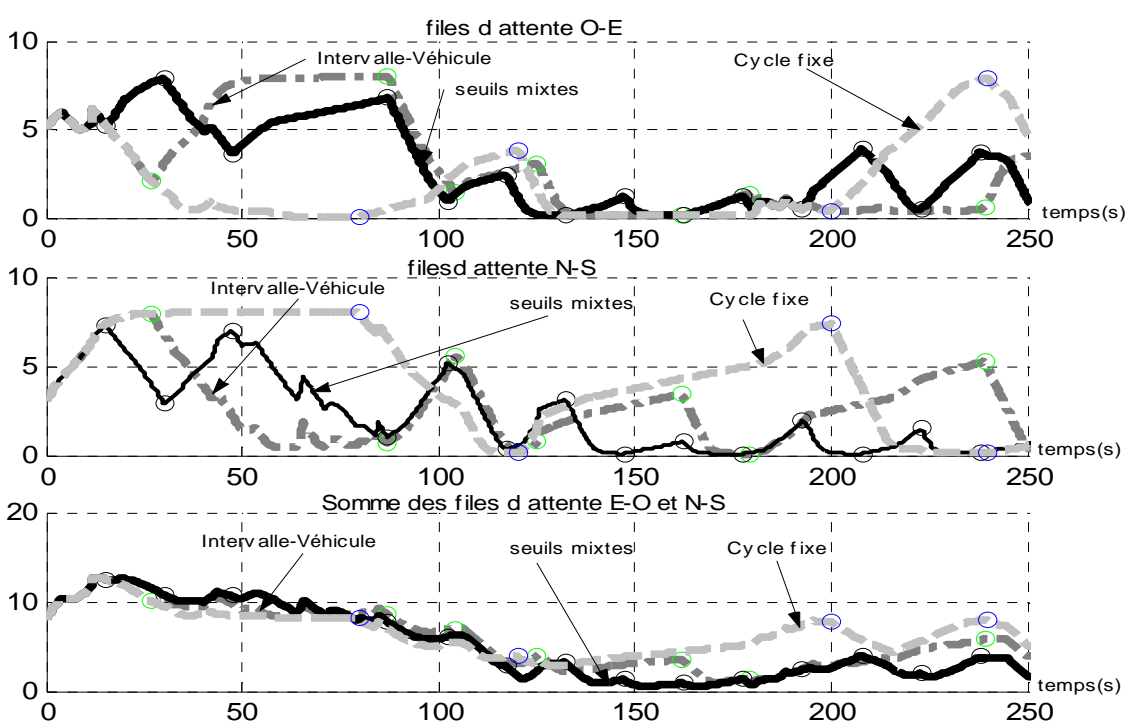

Figure 22. Evolution des files d'attente. 
Les instants de commutation des feux sont présentés par la figure 21. Cette fois encore les profils de commutation de feux sont différents pour les trois commandes. En particulier, la commande à rupture de seuils mixtes présente des durées de vert variables et dépendantes des conditions de trafic sur les voies. La figure 22 présente l'évolution des files d'attente pour les trois commandes. Cette figure montre que, sur cet exemple, les commandes à rupture de seuils mixtes et intervalle - véhicules semblent équivalentes au moins jusqu'à l'instant 140s, instant où la commande à rupture de seuils prend l'avantage.

Cependant, le rapport $\frac{\text { file d'attente pendant le rouge }}{\text { capacité maximale du carrefour }}$ (figure 23) montre que la commande à rupture de seuils est plus performante que ses deux concurrentes et permet, là encore, de minimiser le temps d'attente d'un véhicule au carrefour.

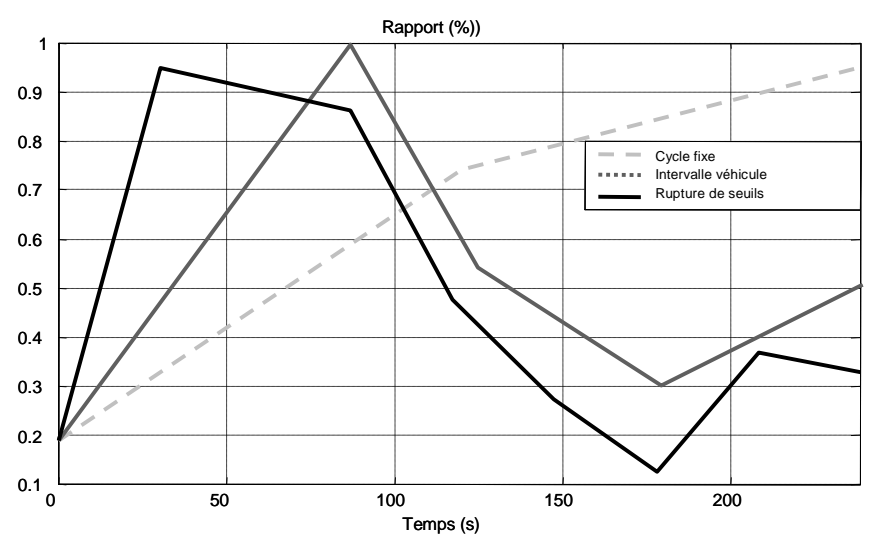

Figure 23. Ratio files d'attente / capacité pendant le rouge.
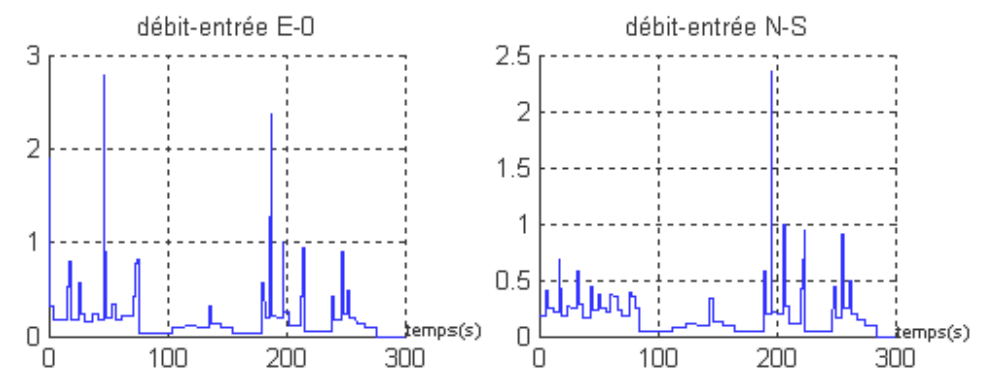

Figure 24. Profils des arrivées de véhicules. 
Les intervalles de temps séparant l'arrivée de deux véhicules successifs sur les voies $(O-E)$ et $(N-S)$ pour un troisième exemple sont présentés par la figure 24 . Le trafic est cette fois caractérisé par un flux plus dense sur la voie $(N-S)$ que sur la voie $(O-E)$.
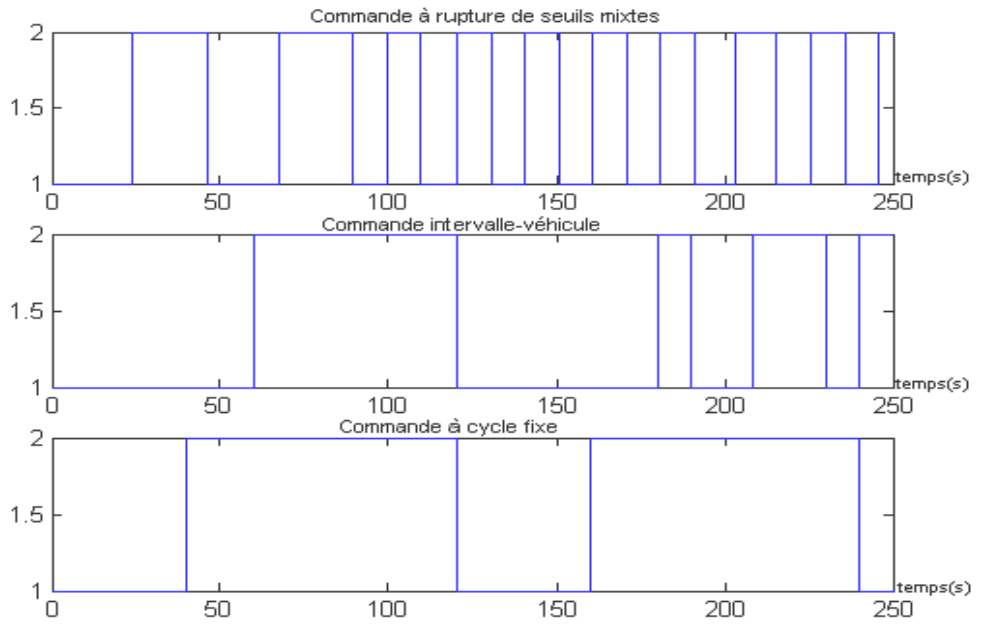

Figure 25. Séquences de commutation des feux.
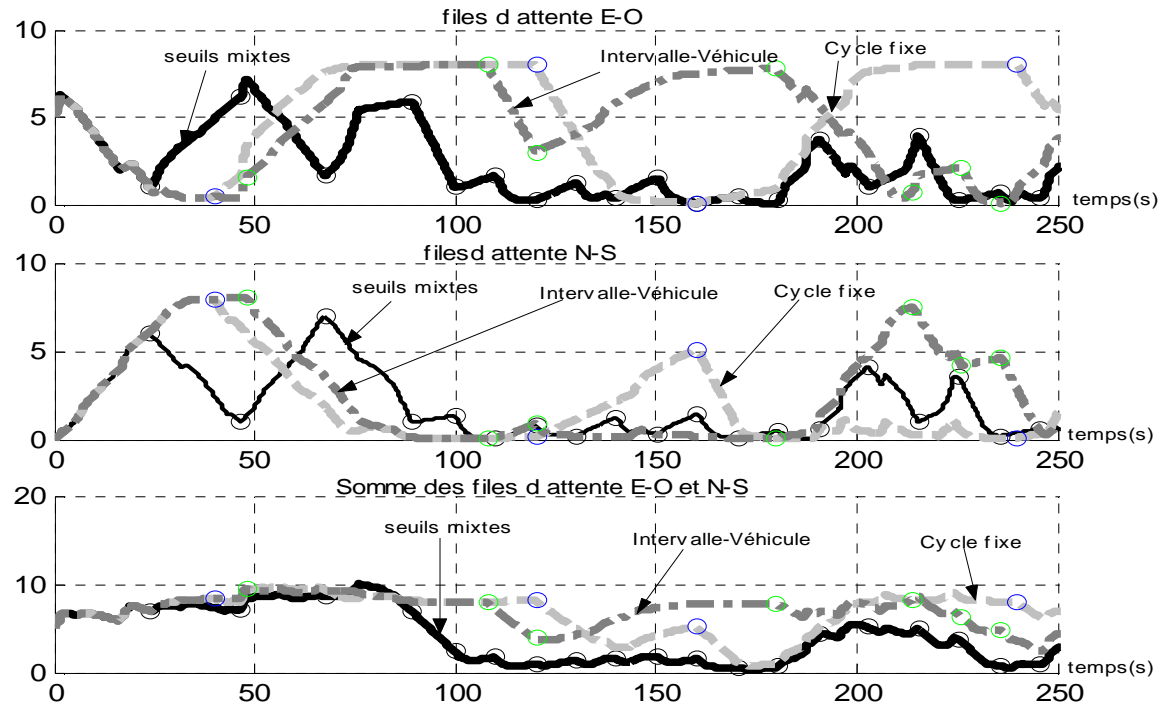

Figure 26. Evolution des files d'attente. 
Les instants de commutation des feux sont présentés par la figure 25. Cette fois encore la commande à rupture de seuils mixtes présente des durées de vert très variables et dépendantes des conditions de trafic sur les voies. La figure 26 présente l'évolution des files d'attente pour les trois commandes. On constate que les trois commandes présentent des fonctionnements équivalents jusqu'à l'instant 90s. A partir de cet instant, la commande à rupture de seuils mixtes s'adapte mieux à la situation que ces deux concurrentes.

La figure 27 confirme ce résultat puisqu'à partir de l'instant 110 s, le ratio files d'attente / capacité est meilleur avec la commande à seuils mixtes par rapport aux deux autres commandes.

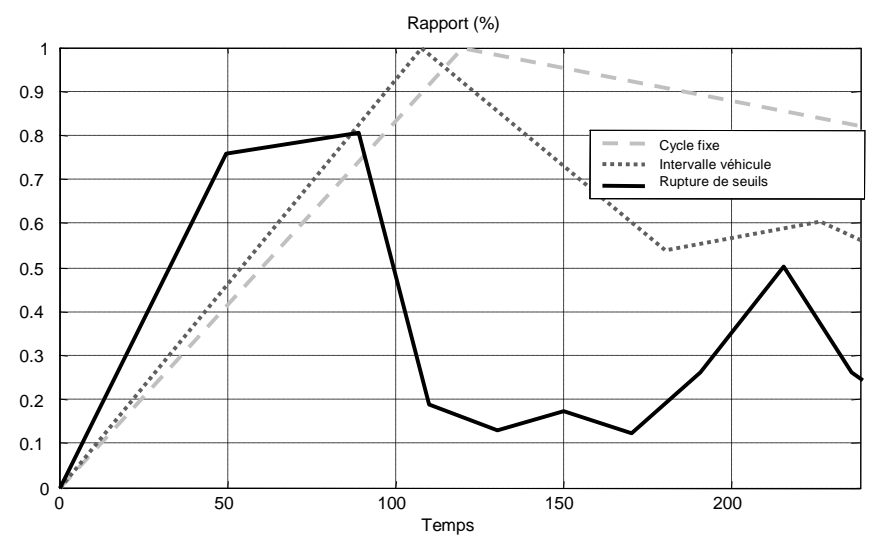

Figure 27. Ratio files d'attente / capacité pendant le rouge.

Les résultats de simulation, Figures 18 à 27, ont permis de montrer l'efficacité de la prise en compte simultanée des seuils bas et hauts dans la commande des feux de carrefour.

\subsection{Commande d'un réseau de plusieurs carrefours}

Pour tirer partie de la commande par rupture de seuils dans la régulation du trafic urbain, nous allons étudier l'évolution du trafic dans le réseau de trois carrefours présenté dans la figure 5. Pour les commandes par rupture de seuil et les commande intervalle - véhicule, nous avons adopté une approche décentralisée sans synchronisation des feux des carrefours (i.e. aucun protocole de communication entre les 3 carrefours n'est utilisé). La commande des feux est locale, c'est-à-dire la commutation des feux est liée uniquement aux fluctuations du nombre de véhicules présents dans chaque carrefour. Dans cette série de simulation, nous allons comparer les performances de la commande par rupture de seuils mixtes par rapport à la commande à cycle fixe. Les longueurs du cycle de feu de $I_{1}, I_{2}$ et $I_{3}$ sont respectivement 60,120 et 75 s partagée uniquement entre le « vert » et le « rouge ». 
La table 6 présente les paramètres du réseau ainsi que les paramètres du modèle RdPH.

\begin{tabular}{|c|c|c|c|c|c|c|c|c|}
\hline & \multicolumn{4}{|c|}{ Paramètres du réseau } & \multicolumn{3}{|c|}{ Paramètres du modèle RdPH } \\
\hline & & $\begin{array}{c}\Delta \\
{[\mathrm{m}]}\end{array}$ & \begin{tabular}{|l}
$v_{\text {free }}$ \\
{$[\mathrm{km} / \mathrm{h}]$}
\end{tabular} & $\begin{array}{c}\text { débit sortant } \\
\text { [véh/s] }\end{array}$ & $\begin{array}{c}c \\
\text { [véh] }\end{array}$ & $\begin{array}{c}q_{\max j+1} \\
{[1 / \mathrm{s}]}\end{array}$ & $\begin{array}{c}a_{j} \\
\text { [véh] }\end{array}$ & $\begin{array}{c}a_{j+1} \\
\text { [véh] }\end{array}$ \\
\hline \multirow[t]{2}{*}{$\overline{I_{l}}$} & $O-E$ & 300 & \multirow{6}{*}{50} & \multirow{6}{*}{0.8} & 60 & 0,046 & 9 & 17.39 \\
\hline & $N-S$ & 150 & & & 30 & 0,092 & 10 & 8.69 \\
\hline \multirow[t]{2}{*}{$I_{2}$} & $O-E$ & 500 & & & 100 & 0,027 & 17.39 & 29.62 \\
\hline & $N-S$ & 375 & & & 75 & 0,037 & 10 & 21.62 \\
\hline \multirow[t]{2}{*}{$I_{3}$} & $O-E$ & 200 & & & 40 & 0,069 & 10 & 11.59 \\
\hline & $N-S$ & 250 & & & 50 & 0,055 & 21.62 & 14.54 \\
\hline
\end{tabular}

Table 6. Paramètres du réseau et du modèle par RdPH.

Les entrées $q_{\max j}$ du modèle par RdPH sont obtenues par inversion des débits d'entrées correspondant aux intervalles de temps inter-véhiculaires distribués selon la loi exponentielle, équation [23], Figure 20.

La décomposition des modèles [13], [14] et [15] selon la fonction $\varphi(t) \in\{0,1\}$ permet d'aboutir à 6 sous systèmes hybrides non linéaires $\left(S_{1}, S_{2}\right)$ similaires à [10] et [11]. La linéarisation de l'ensemble de ces modèles par l'approche multi-modèles fournit 48 équations linéaires affines valides dans leurs domaines respectifs. La figure 28 illustre la séquence d'activation de $\left(S_{1}, S_{2}\right)$ pendant un cycle de feu.

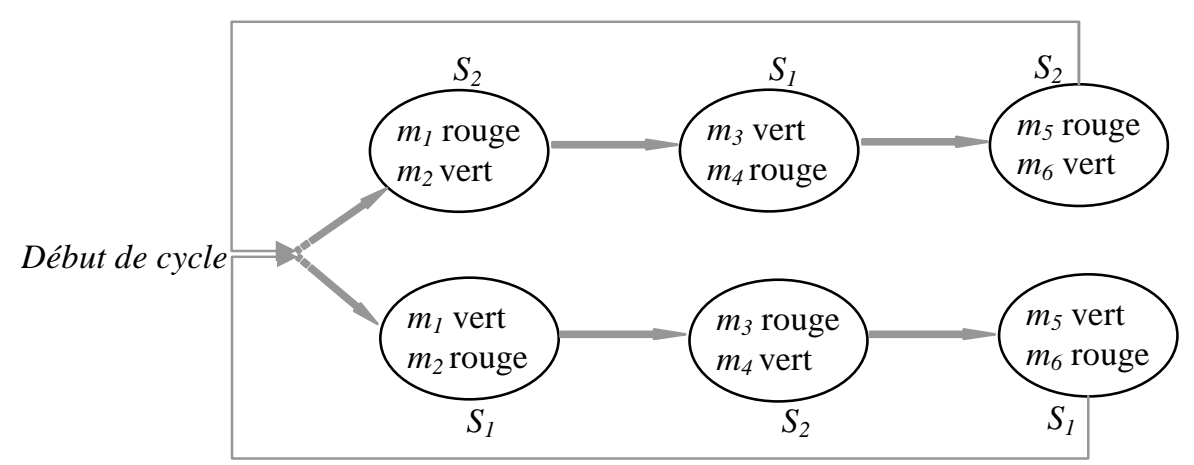

Figure 28. Séquence de commutation des sous systèmes $S 1$ et $S_{2}$ pendant un cycle de feu. 


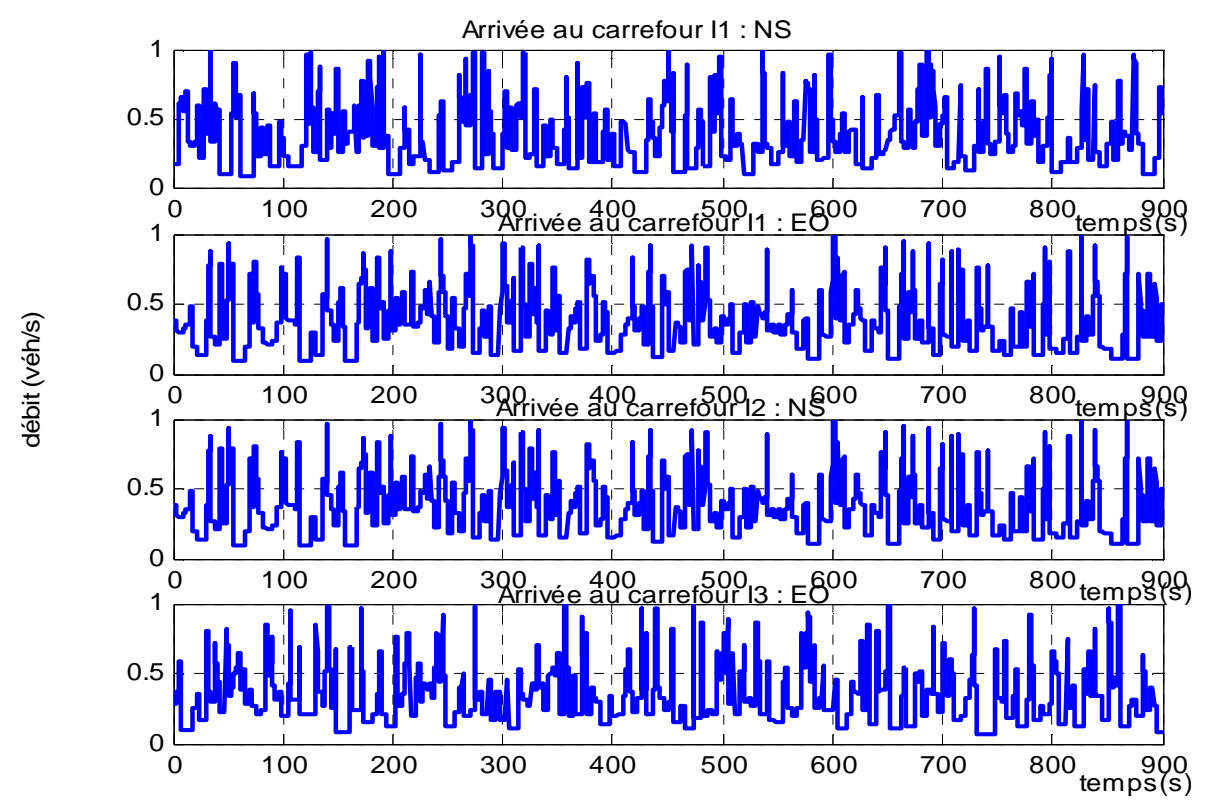

Figure 29. Débits d'entrées des carrefours.

Les durées du «vert minimum » et du «vert maximum » de $I_{1}, I_{2}$ et $I_{3}$ sont respectivement égales à $25,50,30 \mathrm{~s}$ et $30,60,35 \mathrm{~s}$. A l'état initial $t_{0}$ le nombre de véhicules présents dans chaque carrefour est : $m_{1}{ }^{0}=6$ véh., $m_{2}{ }^{0}=5$ véh., $m_{3}{ }^{0}=4$ véh., $m_{4}{ }^{0}=8$ véh., $m_{5}{ }^{0}=0$ véh., $m_{6}{ }^{0}=7$ véh. Le feu est supposé « vert » sur la voie $(O-E)$ du carrefour $I_{1}$ et $I_{3}$, et « rouge » sur celle du carrefour $I_{2}$. 

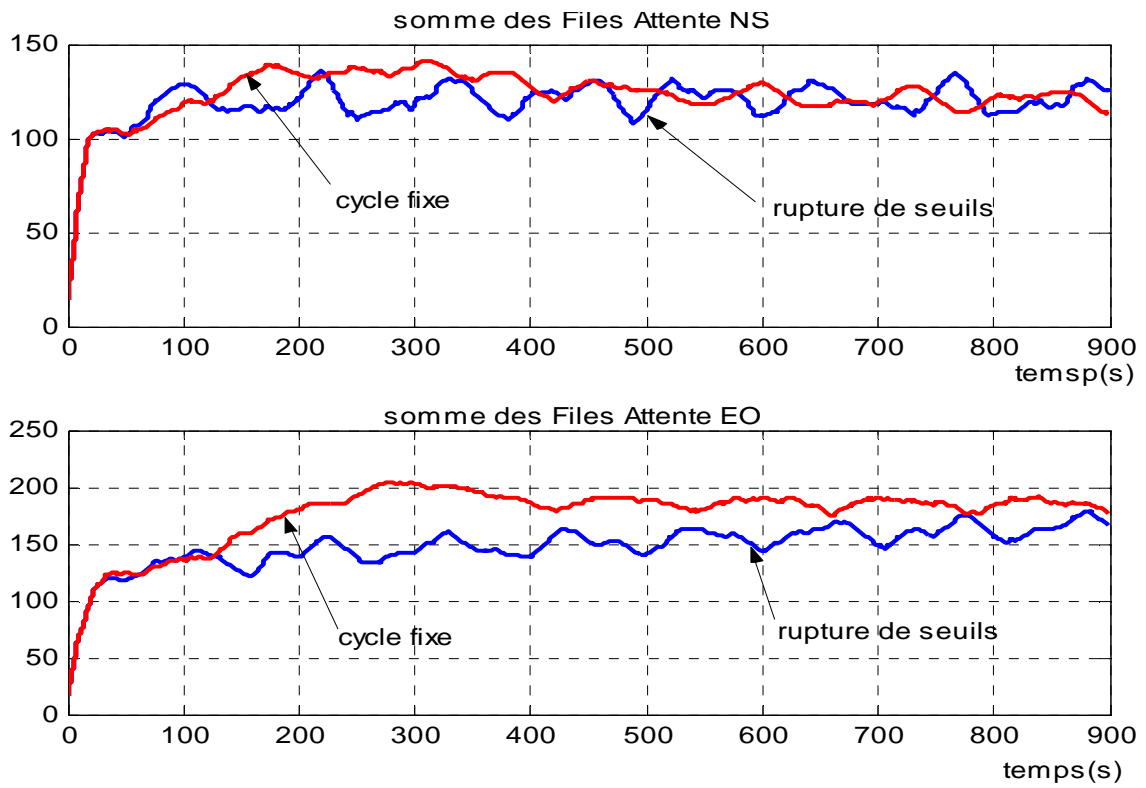

Figure 30. Sommes des files d'attente.

L'examen des courbes des figures 29 et 30 montre que la formation des files d'attente se fait au niveau des voies d'entrées au réseau à savoir les voies O-E du carrefour $\mathrm{I}_{1}$ et $\mathrm{I}_{3}$ et les voies N-S du carrefour $\mathrm{I}_{1}$ et $\mathrm{I}_{2}$. En revanche, les files d'attente au niveau des jonctions intermédiaires (voie O-E de $\mathrm{I}_{1}$ et N-S de $\mathrm{I}_{3}$ ) sont variables (accumulation et dégagement de véhicules). L'analyse des courbes correspondantes montre que la commande par rupture de seuils mixtes permet d'obtenir de bonnes performances comparativement à la commande à cycle fixe en minimisant les longueurs des files d'attente (cf. figure 30).

On peut donc constater que la gestion du flux de véhicules au niveau des jonctions de liaisons entre les carrefours est particulièrement sensible au type de commande appliqué aux feux tricolores.

De manière générale, la commande par rupture de seuils minimise las attentes de véhicules au réseau. Toutefois, l'application de cette commande d'une façon décentralisée reste loin d'une stratégie optimale permettant de réduire significativement les formations des files d'attente et particulièrement au niveau des entrées au réseau.

\section{Conclusions}

Dans cet article, nous avons présenté une approche de commande événementielle dédiée à la régulation des feux de carrefour. Après avoir modélisé la dynamique du trafic (évolution continue des files d'attente et comportement discret des feux) par 
un réseau de Petri hybride, nous avons proposé un système de commande des feux basé sur une approche multi - modèles. La commande obtenue a été simulée et comparée par rapport aux commandes par cycle fixe et intervalle - véhicule, pour un carrefour isolé. Nous avons ensuite élargi cette approche de régulation du trafic urbain à la gestion des flux de véhicules dans un réseau de trois carrefours où la commande par rupture de seuils a été appliquée de manière décentralisée.

Les avantages principaux de la méthode proposée sont: (1) la décomposition modulaire du modèle qui permet d'étendre les résultats à des réseaux de grande dimension à l'échelle d'agglomérations, (2) l'utilisation directe des paramètres du modèle dans la stratégie de commande qui permet une mise en œuvre aisée sans recourir à une étude statistique de la fréquentation du réseau, (3) l'implémentation est facilitée par l'utilisation d'outils de modélisation adaptés à la programmation avec des automates industriels.

Malgré ces avantages, les simulations réalisées ont montré les limites de la commande décentralisée. Il nous parait nécessaire d'étudier la mise en œuvre d'une supervision permettant d'assurer un niveau minimal de coordination entre les différents carrefours d'un même réseau. Dans nos travaux futurs, nous allons prendre en compte les différents mouvements de circulation de véhicules dans un carrefour à feux, à savoir les tourne à gauche et à droite. Il sera également intéressant d'intégrer l'algorithme de commande par rupture de seuils dans le modèle RdPH en le modélisant par un RdP. Ensuite nous mettrons l'accent sur la régulation hiérarchisée d'un réseau de plusieurs carrefours en utilisant un protocole de communication entre les carrefours à feux.

\section{Bibiliographie}

Antsaklis P.J and Koutsoukos X. D, «Hybrid Systems Control », Encyclopedia of Physical Science and Technology, 3rd edition, Academic Press, vol. 7, 2002, p. 445-458.

Boillot F., Midenet S., Pierrelée J.C., «Real-life CRONOS evaluation », Proc. Of the 10th IEE International Conference on Road Traffic Information and Control, $\mathrm{n}^{\circ} 472,2000, \mathrm{p}$. 182-186.

David R., Alla H., Du grafcet aux réseaux de Petri, Paris, Editions Hermès, 1992.

Di Cesare F., Kulp P.T., Gile M., List G., «The application of Petri nets to the Modelling, Analysis and Control of Intelligent Urban Traffic Networks », Proc. of the APN'94, Robert Valette, Zaragoza, Spain, 1994, p. 2-15.

Di Febrarro A., Giglio D., Sacco N., «Urban traffic control structure based on hybrid Petri nets », IEEE Transactions on Intelligent Transportation systems, vol. 5, n ${ }^{\circ} 4,2004$, p. 224-237.

Gallego J.L., Nouvelles approches pour la commande des carrefours, thèse de doctorat, Ecole nationale supérieure de l'aéronautique et de l'espace, 1996.

Gartner N.H., Tarnoff P.J., Andrews C.M., « Evaluation of the Optimized Policies for Adaptive Control (OPAC) Strategy », Transportation Research Record, $\mathrm{n}^{\circ}$ 1324, 1991, p.105-114. 
Hansen B.G., Martin P.T., Perrin H.J., «SCOOT real-time adaptive control in a CORSIM simulation environment », Transportation Research Record, $\mathrm{n}^{\circ}$ 1727, 2000, p.27-31.

Henry J.J., "The PRODYN Real Time Traffic Algorithme », $4^{\text {th }}$ IFAC/IFIP/IFORS International Conference on Control in Transportation Systems, 1983, p. 307-311.

Hunt P.B., Robertson D.I., Bretherton R.D., Winton R.I., «SCOOT: A Traffic Responsive Method of Coordinating Signals », Transport and Road Research, Laboratory Report $n^{\circ}$ LR1014, TRRL, Crowthorne, UK, 1981.

Jensen K., Coloured Petri Nets. Basic concepts, analysis method and practical use, vol. 1, 1992, EATC monographs on Theoretical Computer Science, Springer Verlag.

Khoudour L., Lesort J.B., Farges J.L, «PRODYN - Three Years of Trials in the ZELT Experimental Zone», Recherche-Transports-Sécurité, English Issue, Special Traffic Management, 1991, p.89-98.

Le Bail J., Alla H., David R., «Hybrid Petri nets», Proc. of the European Control Conference, 1991, Grenoble, France, p. 1472-1477.

Lefebvre D., Leclercq E., Druaux F., Thomas P., «Commande des flux dans les réseaux de Petri continus par propagation du gradient», Conférence Internationale Francophone d'Automatique (CIFA), Douz, Tunisie, 2004, CD-Rom.

Lei J., Ozguner U., «Decentralized hybrid intersection control», Proc. Of the 40th IEEE $C D C, 2001$, p.1237-1242.

Nagel K., «Simplified Cellular Automaton Model for City Traffic », Physical review E, vol. 58, n02, 1998, pp. 1286-1295.

Papageorgiou M., Schmidt G., « Freeway Traffic Modelling », Encyclopaedia of Traffic and Transportation Systems, Editor Papageorgiou M., 1991, pp. 162-167.

Papageorgiou M., « Some Remarks on Macroscopic Traffic Flow Modelling », Transportation Research A, vol.32, $\mathrm{n}^{\circ}$ 5, 1997, pp. 323-329.

Papagiorgiou M., Diakaki C., Dinopoulou V., Kotsialos A., Wang Y., «Review of road traffic control strategies », Proc. of the IEEE, vol. 91, n 12, 2003, p. 2043-2067.

Payne H.J., « Models of Freeway Traffic and Control», Math. Models Publ. Sys. Simul. Council Proc., $\mathrm{n}^{\circ}$ 28, 1971, pp.51-56.

Sims A.G., Finaly A.B., «SCATS: Splits and Offsets Simplified SAS », Aust. Road Research Board. Proc. vol. 4, n¹2, 1984, p. 17-33.

Taylor W.C., Abdel-Rahim A.S., Incident Management Under SCAT Adaptive Control System FAST-TRAC Phase III Deliverable \#11, Final Report On Incident Management Under SCATS adaptive Control System EECS-ITS LAB-FT98-084, 1998, p.01-20.

Tolba C., Lefebvre D., Thomas P., ElMoudni A., «Continuous Petri nets models for the analysis of traffic urban networks», Proc. of the IEEE Systems, Man, and Cybernetics Conference, Tucson, Arizona, USA, 2001, p.1323-1328.

Tolba C., Lefebvre D., Thomas P., ElMoudni A., « Continuous Petri nets for the microscopic modeling of traffic flow», Proc. of Summer Computer Simulation Conference (SCSC'02), San Diego, California, USA, 2002, CD-Rom. 
Tolba C., Lefebvre D., Thomas P., ElMoudni A., «Performance evaluation of the traffic control in a single crossroad by Petri nets», Proc. of the $9^{\text {th }}$ IEEE International Conference on Emerging Technologies and Factory Automation (ETFA'03), vol. 2, Lisbon, Portugal, 2003, p. 157-160.

Tolba C., Lefebvre D., Thomas P., ElMoudni A., «Approche multi-modèles pour la commande des feux de traffic », Conférence Internationale Francophone d'Automatique (CIFA), Douz, Tunisie, 2004, CD-Rom.

Tolba C., Contribution à l'utilisation des réseaux de Petri pour la modélisation et la régulation du trafic urbain et interurbain, thèse de doctorat, Université de Technologie de Belfort Montbéliard, 2004.

Wang H., List G.F., Di Cesare F., «Modelling and Evaluation of Traffic Signal Control Using Timed Petri Nets », CESA, vol. 2, Le Touquet, France ,1993, p. 180-185. 